\title{
ESTUDIOS SOBRE LA ESTRUCTURA DEL ADVERBIO PRONOMINAL INDOEUROPEO
}

This paper is an analysis of the inner structure of Indoeuropean pronominal adverbs, including the simplest suffixed forms as well as compounds and derived forms; the authoress aims also at an elucidation of the syntagmatic relations of their constituents and a reconstruction of the different stages of their history from the point of view of word formation, morphonology and morphosyntax, in order to arrive at original systems sometimes showing a very different situation from the traditionally accepted.

\section{Definición}

1.1. El adv. ide. presenta, como obstáculo para su clasificación, previo a su definición propiamente dicha, su construcción sobre los dos grandes tipos que configuran el léxico ide.: me refiero a las raices nominales y a las pronominal-adverbiales. La dificultad consiste, por un lado, en atribuir a un adv. o conjunto de ellos origen en uno de los tipos, lo que, a su vez, condiciona la interpretación de sus variantes, y por otro, en que la existencia misma de algunas de estas raices depende de la consideración que se les dé a los advs. que al parecer la integran.

1.2. En segundo lugar, el adv. precisa para su estudio de una restricción sincrónica del material. En concreto, se admite que lo que en fases muy antiguas ha debido de pertenecer a una sola clase léxica, se reparte en las lenguas ide. entre advs., conjs. y preps. Aquí se puede provisionalmente añadir que también ciertos casos pronominales pueden ser clasificaciones tardías de antiguos advs.

1.3. Por último, se debe atender a lo que caracteriza al antiguo adv. como tal y lo distingue sincrónicamente de las demás clases. En lo que toca a las raices pronominales, la diferencia es cualitativa y cuantitativa a un tiempo. Pues no considero adv. un elemento puro, con independencia de su estatuto funcional. Un adv. será una forma al menos 
bimembre, que porta sobre la raíz un $2 .^{\circ}$ constituyente, un morfema flexivo ${ }^{\text {. }}$.

\section{II. ¿EXISTIÓ UN ADVERBIO INDOEUROPEO?}

2.1. La opinión general, la que parece sobrevivir con más firmeza al curso del tiempo, y también la que se basa principalmente en datos de otras categorías, sostiene que la categoría de adv. era desconocida en la protolengua. Sostiene también que esta clase se ha formado paulatinamente a partir de los residuos de sust. y pron., que, según las lenguas o grupos lingüísticos, perdían casos en número variable por el procedimiento del sincretismo. Esta hipótesis, cuya validez no puede negarse por completo ni para todos los casos, tropieza con diversos inconvenientes ${ }^{2}$.

2.1.1. El primero es de carácter funcional. No es fácil imaginar una lengua sin advs., y menos una que ha desarrollado la complejidad morfológica que conoció el ide., incluso visto desde las perspectivas más reduccionistas. No olvidemos que el adv. es una de las formas básicas de llamar la atención sobre la realidad. Podrá objetarse sin duda que esta fase de la lengua podría tener advs., pero que éstos constituyen a su vez fosilizaciones de sistemas casuales pre-proto-ide. perdidos hace muchos milenios. La respuesta es que, extraído para la protolengua un sistema adverbial que no corresponde al sistema casual conocido, es indiferente, por indeductible, su estatuto sintáctico adverbial o pronominal/nominal antes de la protolengua misma, y que sólo cuenta para mí su posible disgregación de otras categorías del material ide. tal como lo conocemos.

2.1.2. El segundo escollo toca a la morfología de la terminación adverbial. Es muy frecuente que entre los advs. encontremos morfemas en final que no se corresponden con ningún caso de la flexión en ninguna lengua, o en la familia concreta que se está estudiando.

2.1.3. En multitud de ocasiones, el adv. pertenece a una raíz pronominal que no aparece en todo un grupo lingüístico más que en advs. Así sucede, en todo el ide., con ${ }^{*} d e-{ }^{*}{ }^{*} d o-y^{*}$ dhe- $/{ }^{*}$ dho-.

2.1.4. Por último, hay que atender a las dificultades teóricas que plantea el proceso evolutivo de adverbialización de casos de la flexión,

1 Flexivo se entiende por oposición a derivacional, como segmento de contenido sintáctico, sin que ello implique pertenencia a un paradigma. Flexivo y derivacional se entienden sincrónicamente.

2 Aunque a veces se juzgan variaciones cuantitativas como ${ }^{*}-\left.e\right|^{*}-\bar{e}$ como un fenómeno aleatorio. Cf. F. R. Adrados, Lingüistica indoeuropea, Madrid 1975. 
cuando esta sencilla regla explicativa se extiende a todos los advs. ilimitadamente. El problema es diverso en el caso de los advs. pronominales y en el de los nominales. La dispersión formal de los elementos que aparecen en el final de un adv. pronominal exige, como es obvio, recurrir a la práctica totalidad de los casos de la flexión para identificarlos. Pero una crítica detallada de la evolución propugnada revela a veces el sinsentido de este método. La primera objeción se refiere a una contradicción en el sistema clásico reconstruido. Por un lado, se parte de un ide. de ocho casos, o sea de máxima diferenciación interna del sistema flexivo nominal y pronominal. Este sistema justifica la pluralidad de finales adverbiales, y en muchos casos esta pluralidad justifica a su vez ese sistema flexivo nominal / pronominal. Pero además, esta misma abundancia de terminaciones casuales en los casos oblicuos hace inverosímil la fosilización de los casos rectos. Un caso aberrante es el del lat., donde aparecen para una misma raiz pronominal *ne-/*no- advs. que representan todos los tipos de ac. conocidos (jen la flexión nominal!) y en todos los géneros, como nam, num, enim, nem-pe. Aquí el método clásico procede contra toda verosimilitud al suponer una flexión pronominal que a) se ha perdido b) lo ha hecho no sin fosilizar previamente todos sus acs. c) lo ha conseguido sin fosilizar los numerosos casos modales y locales de los que teóricamente disponia.

En segundo lugar, no es directamente admisible hablar de fosilización de casos rectos masc. y fem. de la flexión pronominal. Sus defensores ponen como ejemplo los advs. modales que proceden sin duda de susts. y adjs. en su forma de nom.-ac. Sin embargo, la relación ofrece una dificultad insalvable. Un adj. o sustantivo en función predicativa es, en principio, funcionalmente ambiguo, porque dicha función sintáctica, por sus condiciones de subcategorización, permite ser ocupada por ambas clases de palabras, adj. y adv. Por tanto, en el caso de adj. y sustantivo, la adverbialización (aun suponiendo que la haya y que no hablemos de una cuestión sintáctica sincrónica), depende de un contenido semántico adverbializable. En el caso p. ej. de '(a) éste, (a) ésta' > 'así', no hay contenido semántico que adverbializar y la evolución es caprichosa: si decimos 'el árbol es éste' lo estamos señalando y oponiendo el lugar que ocupa al de otros, no describiendo. No hay en cambio grandes dificultades para suponer que un nom.-ac. neutro equivale sincrónicamente a un adv. 'así'. Si decimos 'el árbol es esto', 'esto' resume un número de características visibles aunque no especificadas, como 'verde, alto, vegetal', que pueden resumirse por un adv. equivalente 'asi'. Y sin embargo no hay, en lat. ni en las otras lenguas, un adv. ${ }^{* *}$ nod, ni ${ }^{* *}$ kod, ${ }^{* *}$ dhod o **dod. 


\section{MÉTOdO Y CORRESPONDENCIA}

3.1. Un punto llama la atención en la morfosintaxis del ide. tal como viene aplicándose tradicionalmente $\mathrm{y}$, por tanto, también al adv. $\mathrm{Y}$ es que, con excepciones recientes (Villar, Shields, Schmalstieg) se hace de los casos compartimentos estancos y aislados entre sí. De ello se deduce que los advs. no son nunca variantes fonotácticas no regularizadas de un solo elemento inicial, sino elementos separados remitibles cada uno sólo a su caso correspondiente. Sin embargo, de la observación del adv. ide. se deduce una situación muy diferente. Si prescindimos de la comparación directa de formas idénticas en diversas lenguas y nos fijamos en los subsistemas de cada lengua o grupo, el resultado es una serie de constelaciones cuyos miembros: a) no pertenecen al sistema casual de ninguna lengua, o no al de ese grupo lingüístico; b) pueden pertenecer formalmente al sistema casual reconstruido tradicionalmente sólo si se niegan los supuestos evolutivos detallados más arriba (2.1.4); c) pueden ser correspondencias si se admite para ellos un étimo común, fonéticamente distinto al que permite reconstruir cada uno de ellos. Así, ${ }^{*}-\bar{e}$ y ${ }^{*}-e i$ nos permiten suponer una forma previa ${ }^{*}-e H_{1}-i$, salvo que nos ciñamos al apriorismo de que siempre han sido morfemas distintos y por ello deben haber sido secuencias fónicas distintas.

3.2. Paso, pues, a definir las circunstancias a las que obedece para mí la inclusión de un elemento en una determinada constelación, y de la constelación a su vez en un adv. único:

a) Pertenencia a un mismo núcleo temático. Es decir, que las formas que pueden haber conocido un único étimo comparten necesariamente una raiz, en este caso pronominal-adverbial.

b) Posesión de un morfema adverbial común. La aplicación del método intralingüístico relega la comparación propiamente dicha a un estadio de la investigación posterior a la comprensión de las conexiones entre elementos visiblemente relacionados de una misma lengua. Conforme a esto, se consideran como hipotéticamente ligados aquellos elementos reductibles a uno solo en fases anteriores, del que en la actualidad representan variantes morfonológicas, elementos que, conforme a lo dicho, no pueden por su forma haber pertenecido a la flexión pronominal tal como la conocemos.

c) Isofuncionalidad. La no diversidad funcional de finales adverbiales retrotraídos tradicionalmente a casos que expresan nociones varias sirve en ocasiones de confirmación adicional de su identidad originaria. 


\section{AdVERBios PRONOMINALES BÁSICOS}

\subsection{Morfologia}

Como ya he adelantado, el adv. pronominal es un elemento complejo que consta de una base deíctica a la que se añade un categorizador propiamente adverbial. Éste es el que distingue formalmente un adv. de un pron., y el que nos permite aislar una serie de elementos, como veremos muy repetidos, que no son terminaciones pronominales. Ahora bien, precisemos qué hace suponer que determinados finales no pertenecen a la órbita de la flexión pronominal.

Desde una perspectiva sincrónica, resulta evidente la no pronominalidad funcional de preverbios, preps. y conjs., no así la de los advs. pronominales de carácter modal, local, etc. En este último caso me baso para decidir en varios factores decisivos, como son: el hecho de que una forma no acompañe nunca a un núcleo nominal como atributo; el hecho de que una forma no se refiera anafórica o catafóricamente a nada que no sea una acción o asunto, para los que se pueda prescindir de la distinción de género (con ello, al. womit, etc.).

Diacrónicamente pueden excluirse de consideración los casos en que el adv. procede claramente de un caso fosilizado en el curso de la evolución de un grupo lingüístico, de manera que en una de las lenguas que lo componen es adv. y en las demás es aún loc., instr., etc., y también los casos en que estamos ante el uso adverbial de un caso oblicuo que sincrónicamente pertenece a la flexión.

Delimitada la serie de elementos que se oponen a sustantivo y verbo en portar raíz deíctica, y que se oponen al pron. en llevar un final no pronominal, queda establecer las relaciones entre los diversos finales. Éstos pueden ser reducidos a un común denominador en la medida de lo posible fonéticamente. Y a ello contribuye además su eventual identidad funcional, en la medida en que no proceda claramente de una uniformización causada por la raíz. Más adelante hablaré de las dificultades que ocasiona la interferencia entre el significado deíctico y el sintáctico en ciertos grupos.

Me ceñiré a las raíces ${ }^{*} k^{4} e-/{ }^{*} k^{*} o-,{ }^{*} t e-/{ }^{*} t o-,{ }^{*} i e-/{ }^{*} i o-,{ }^{*} k e-/{ }^{*} k o-$, *ghe-/*gho-, ${ }^{*}$ de- $/{ }^{*}$ do-,${ }^{*}$ dhe- $/{ }^{*}$ dho-, ${ }^{*}$ ne- $/{ }^{*}$ no-, ${ }^{*} s(u) e-/{ }^{*} s(u) o-{ }^{3}$. Y empe-

${ }^{3}$ Dejo de lado *bhe-/*bho-, dado su peculiar estatuto de desinencia aglutinada en varias lenguas. A veces se postulan raícen pron. como *eue- (con $e$ - optativa, J. Mendoza, «Las clases de palabras en el indoeuropeo flexional y en el proto-indoeuropeo, RSEL 5, 1975, pp. 149-63; Adrados, op. cit., p. 850), o *epe- (Adrados, p. 851) que yo estimo nominales, respectivamente ${ }^{*} \mathrm{H}_{2}$ eu- y ${ }^{*} \mathrm{H}_{1}$ ep-. No veo motivo para reconstruir ${ }^{*}$ ete-, ${ }^{*} e n e-$ en ide. ${ }^{*} H_{1} e t i,{ }^{*} H_{1} e n(i)$ (Adrados, pp. 850-52). 
zaré por poner ejemplos de todo el "grupo residual», es decir de todo el conjunto de formas de las que sólo se sabe lo que no son (nominales o pronominales) y en las que trataré de establecer un orden. Lo haré en dos direcciones: 1) por una parte, formas que aparecen para un grupo lingüístico y una raíz (resumen de las raíces más relevantes, mejor documentadas y menos susceptibles de haber sufrido la acción de la analogía). 2) Por otra, formas al parecer idénticas sobre distintas raíces y en un corte transversal que abarque las diferentes lenguas ide. Sin embargo, como dije arriba, el sistema que nos lleva a la forma primigenia se extrae sobre todo por medio de la comparación intralingüística. Por ello, la función del paso 2) consiste sobre todo en poner de manifiesto que la no-pronominalidad que atribuyo a un final adverbial está muy extendida para una o más raíces. Como he dicho, prescindo aquí de la coincidencia formal posible con casos masc. y fem., de cuya irrelevancia trato bajo 2.1.4.

\subsubsection{Finales vocálicos}

Clasificación por temas:

${ }^{*} k^{u} e-/{ }^{*} k^{u} o-$ :

gr. $\pi \eta, \pi \omega, \pi \circ \tilde{\imath}, \pi \circ \tilde{u}, \pi \varepsilon \tilde{\imath}, \pi \tilde{-}-\varsigma$ (advs. locales) / lat. qui 'como', qui-r-qui-r 'dondequiera que', quō '¿adónde?', cū-r ‘ipor qué?' / germ. *hwē, ${ }^{*} h w \bar{e}_{2},{ }^{*} h w \bar{i},{ }^{*} h w \bar{u},{ }^{*} h w \bar{o}$ (advs. interr. modales) / indo-ir. ai. ${ }^{*} k \bar{u}, k u v a$ ‘dónde?', kava-, kva, ko- prefs. peyorativos y cuantitativos de origen modal, av. 'kü(-), kva, kava- ‘dónde?', kà ‘¿cómo?', ap. ada-kaiy 'por entonces', $k \bar{a}$ (partíc. generalizadora), av. ci- (?) ‘cómo?', ap. ciya-karam '¿cómo de grande?', sogd. $k w$ 'dónde, hacia' / esl. rus., che. $\check{c} i$ 'tanto si como', aesl. č̌ 'sin embargo', rus. - $\check{c} a$ (refuerzo), pol., che., eslovaco, arus. $k u$ (prep. de dat.) 'hacia', rus. arus. $c i$ 'si... como si', aesl. $k \check{u}$, rus. $k, k o$, s.-cr. $k a ̈$ prep. de dat. 'hacia', pol., ucr. $-k$ (refuerzo), aesl. $n e-k \breve{u}-l i$ 'que (+ comp.)', aesl. rus. s.-cr. $-k a$, rus. (-)ko(-), ucr., che. $-k y$ (refuerzo) / bált. lit. keĩ 'como', kaĩ 'como, ¿cómo?, cuando', -ki (refuerzo de impvo.), ku-r̃ '¿(a)dónde?', let. kei (?), kai 'como', kaû-t 'que' (conj.), 'si' (desiderativo), ku-r ‘(a)dónde?', aprus. key (?), quei ‘donde, ¿dónde?', kay, kāi 'como, que, ¿cómo?' / toc. A kus, B kuse = ¿quién, qué?' (base de tema pron.), A kupre, B kuri/krui 'si', B kà 'por qué', $k a ̄ t u$ 'porque', B $t(a) k \bar{a}$ 'en efecto', yak(a), ykäk 'aún, entretanto' / anat. het., pal., lid. $k u$ (refuerzo de advs.), het. kussan '¿cuándo?' y tal vez luv. jer. ku-man; luv. jer. (Karatepe) $R E L-i$ 'y' ( $<^{*}{ }^{*} k^{u}[e] i /{ }^{*} k^{u} i$ ? $)$, quizá 
het. ta-kiya 'en otro lado' / celt. galés py 'hacia', airl. co, cu 'hasta' 4 , galés cw 'dónde', quizá celtib. [tuiniku]-kuei [x1] / alb. kūr '¿cuándo?', ku ‘dónde?' / afrig. key pospuesto a verbos y sust. ${ }^{5}$

$$
\text { *ne-/*no-: }
$$

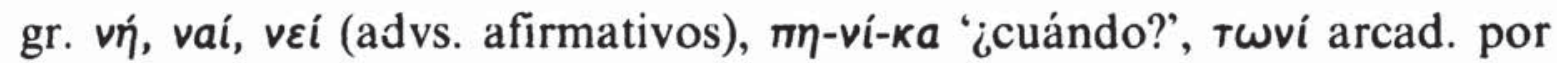
тои̃ $\delta \varepsilon$, i-va 'para que, donde' / lat. nēe, nae 'sí, en efecto' / germ. *-né, ${ }^{*}-n i,{ }^{*}-n a,{ }^{*}-n a i,{ }^{*}-n i$ (?) (suf. locales) / indo-ir. ap. ya-naiy '(a)donde' / frig. vı, en los vı 'quienquiera que', aıvı 'o', etc. / toc. A -ne / B -nai (refuerzos), B ña-ke 'ahora', ñerwe 'hoy' $\left(<{ }^{*} n i-\right) /$ anat. het. ka-ni $\left(<{ }^{*} n[e] i\right), \operatorname{kan}(a)$ 'aquí, luv. za-ni / celt. airl. cuin ‘cuándo?, cuando' $\left(<{ }^{*} k^{u} o-n i\right)$, galo poni: airl. cen 'sin' (si < $\left.{ }^{*} k i-n a\right)$.

$$
\text { *ie-/*io-: }
$$

gr. $\eta, \varepsilon l$, oủ (advs. locales) / germ. ${ }^{*} j a u,{ }^{*} j u,{ }^{*} j \bar{u},{ }^{*} j o ̈ r$ (isl. jór), ${ }^{*} j \bar{e},{ }^{*} j \bar{e}_{2}$, *ji, *ja, *jai 'ya, sí, también, pero' / indo-ir. ai. iv-a 'como', av. yä 'porque, como' * $y \bar{o}$ (coordinador de sust.), ap. yā $\bar{a}$ 'tanto tiempo como' / bált. lit. jaĩ, jéi, jeĩ 'si, aunque', jẽ- 'excepto si', ja-g 'que, porque', jaũ 'ya, sí', jù 'sí', let. ja 'sí' je-, jàu 'pues, sí', ju 'pues', (dial.) jòu / esl. aesl. rus. $j u, i$ 'y' (< pesl. $\left.{ }^{*} \tilde{\jmath} l^{*} j \tilde{u} /{ }^{*} j i\right)$, aesl. ja- / toc. B ya-ka, ya-k, $y-k \bar{a} k$ $\left(<{ }^{*} j u\right)$, A yo 'y'.

$$
{ }^{*} k e-/{ }^{*} k o-:
$$

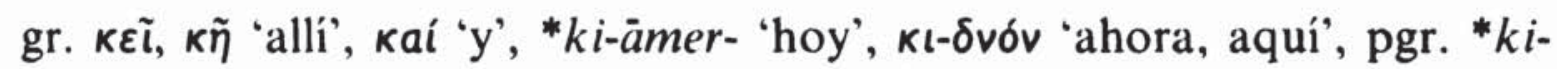
wetes ya en mic. za-we-te 'este año' ( ${ }^{*} k j a \bar{a}$ - por ${ }^{*} k i$-ämer-) / lat. ceu (< $\left.{ }^{*} c e i-u e\right)$ 'como', cè-eter- 'el resto', $-c\left(i<{ }^{*} k i\right.$ ? $) /$ germ. ${ }^{*} h \bar{e}_{2}-r,{ }^{*} h \bar{e}_{2}$ (nom. pron. aing., as.), ${ }^{*} h i-r /{ }^{*} h i-r$ 'aquí', ${ }^{*} h i-s-$ en aing. hislic 'conveniente, del mismo tipo' / indo-ir. sogd. $s^{\prime}-r\left(i<{ }^{*} k \bar{e}-r\right.$ ?) posp. lativa / balt. lit. šè 'aquí', šeĩ-p, šiai-p 'así, si no', let. še, šei, aprus. schai 'aqui' / toc. A širaš 'alrededor' (si < *kei-r-), B $t(a) k \bar{a}, y k a \bar{k}$ (?) / afrig. asi $\left(<{ }^{*} a d-k e i^{6}\right.$, tal vez mejor *-ki) 'aquí', sest 'aquí' / vén. ke 'y' / anat. lic. se 'y', het. ki-nun 'ahora', -kiya en ta-kiya 'en otra parte', kis-san 'así', pal. ki-at 'aquí', het. $k \bar{a}$ 'aquí' / celt. airl. cé, cia 'aquí', ogám. $C I$

${ }^{4}$ Con geminación, cf. Hamp, "Middle Welsh py 'to', bwy-gilydd", BBCS 16, 1956, pp. 281-84.

$5 \mathrm{Cl}$. Brixhe, "Comparaison et langues faiblement documentées: l'exemple du phrygien et ses voyelles longues", La reconstruction des laryngales, 1990, p. 69.

6 I. Diakonoff-V. Neroznak, Phrygian, N. York 1985, p. 93. 
(hoy considerada f.l.), COI, galo kovı (inscr. de Cavaillon; tal vez forma fantasma), $d u-c i$ 'hacia aqui'.

Clasificación por terminaciones (se dan sólo significados de formas no dadas arriba):

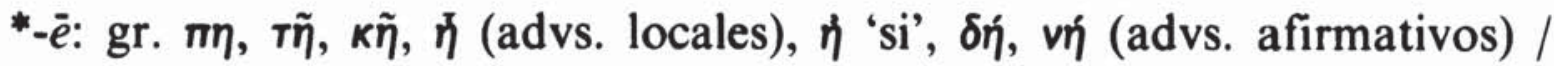

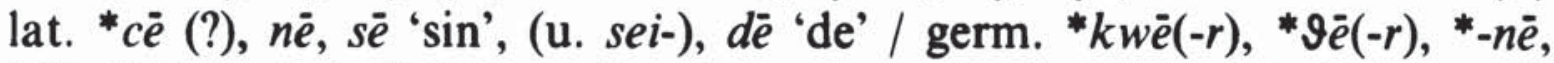
${ }^{*} j \bar{e},{ }^{*} h \bar{e},{ }^{*} s w \bar{e}$ 'asi' (gót. swē, tal vez aaa. sā-r 'pronto' / indo-ir. sogd.

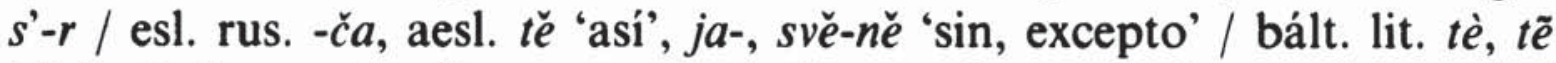
'ahi', jẽ, let. $t e, j e-$, se, aprus. ti-t 'así, o sea', te-r '( \pm )... que' (cpvo.) / vén. $k e$ / toc. A $c a-$ / B $c e(-)$ tema pron. / anat. lic. se / celt. airl. di 'de'.

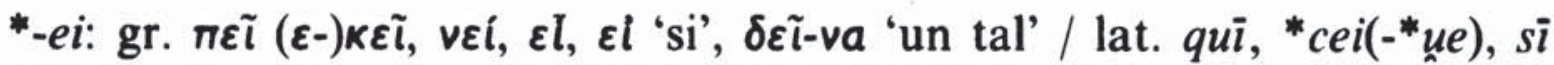
'si', hi-c 'aquí' / germ. * $h w i,{ }^{*} \vartheta \bar{i},{ }^{*} n \bar{i},{ }^{*} \bar{j},{ }^{*} h \bar{i},{ }^{*}$ swi (en an. svi, interj. peyorativa) / indo-ir. ap. -naiy, -kaiy (?) / bált. lit. keĩ, jeĩ, šeĩ, teĩ 'así' / esl. aesl. či, $t i$ 'y', $i$ / toc. A ašši ( $i<$ ats-"tei?) 'acaso', șiraš (< *kei-r-?), si $<{ }^{*}$ suei al. 'her' / vén. .e..i.k. < * ghei-ke?' / frig. key, asi (si *-kei) / anat. het. -kiya, ki-nun, ka-ni luv. jer. $R E L-i$ (?) / celt. airl. cé, cia, celtib. -kuei.

${ }^{*}$-èi: germ. ${ }^{*} h w \bar{e}_{2},{ }^{*} h \bar{e}_{2}-r,{ }^{*} j \bar{e}_{2},{ }^{*} s w \bar{e}_{2}$ (sue. sve- pref. peyorativo, aaa. see 'ahí') / bált. lit. jéi / toc. A ce-, B cai- (tema pron.).

*ai: gr. kal, vaí, al 'si', oal 'si' / lat. nae, o. svai, u. sve 'si' / germ. *jai, *s(w)ai (an. svei, interj. peyorativa, aaa. see, gót. sai “jahí!), *-nai / indo-ir. ap. -naiy, -kaiy, ada-taiy 'después' (si no es el dat. de *tu) / bált. lit. kaĩ, jai, šiaĩ, taĩ 'así' / esl. aesl. če, tě, svě-ně / toc. A te, B tai-su 'así', A -ne, B -nai / frig. al (conj. disyuntiva $<{ }^{*} s[u] a i$ ).

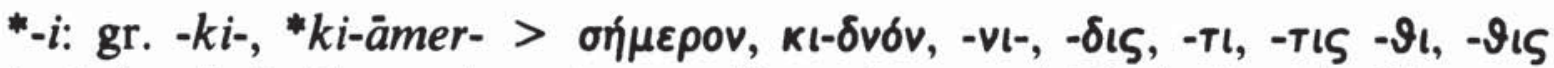
(suf. locales) / lat. qui-r, ci-s, -ne (?), -c / germ. *hi(-s-), *-ni / afrig. sest $\left(\mathrm{si}<{ }^{*} k i-\right)$, nfrig. vi $\left(<{ }^{*} n i /{ }^{*} n i /{ }^{*} n e i\right) /$ bált. lit. $k i /$ anat. het. -kiya, $k i-$ nun, $k a-n i$, luv. jer. $R E L-i$ / celt. airl. cuin, cé, cia, ogám. CI (?), galo du-ci.

*-i: [cf. *-ei para lenguas que identifican ambos resultados] indo-ir. av. ci- (?), ap. ciya-.

${ }^{7}$ M. Lejeune, Manuel de la langue vénète, Heidelberg 1974, p. 100. 
*-oi: [cf. *-ai para lenguas que identifican ambos resultados] gr. поĩ, noı, тo (partíc. encl., si no es el dat. de oú) / airl. ogám. COI, galo *kout.

*-ō: gr. $\pi \omega, \tau \tilde{\omega}, \tilde{\omega}, \tilde{\omega}-\varsigma$ 'asi' / lat. $q u \bar{o}, h \bar{o}-c$ 'hacia aqui', sō-c 'asi', u. surur $\left(<{ }^{*} s[u] \bar{o}-s[u] \bar{o}-r\right)$ 'igualmente' / germ. ${ }^{*} h w \bar{o},{ }^{*} \vartheta_{\bar{o}}$ 'entonces, cuando', $s(w) \bar{o}$ 'asi' / indo-ir. ai. ap. av. $k \bar{a}$, av. $y \bar{a}, t \bar{a}$ / esl. aesl. ja-, $k a$, $t a$ 'así' / bált. lit. kuõ, tuõ (en situación semi-paradigmática!), juõ / toc. B $k \bar{a}, t(a) k \bar{a}$ / alb. te 'en' (?) / anat. het. $k \bar{a}$ 'aquí', tal vez en parte $t a$ (partíc. conectiva) / celt. celtib. (Botorrita $A_{1}$ ) sua 'así'.

*-ou (*-au): gr. пои̃, oũ / lat. cū-r, hü-c / germ. *jau, *פau /indo-ir. ai. ko-, kava-, av. yō /esl. arus. ku, aesl. ju, tu 'alli', su- en su-gubŭ (?) / bált. let. kâ̂-t, lit. jaũ, tau-ciaũ 'sin embargo' / toc. A yo / anat. het. $-k u$.

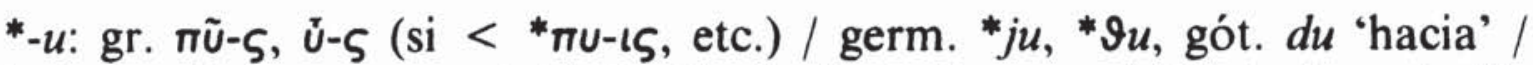
indo-ir. ai. $k v-a, i v-a, t u ́$ / bált. lit. $k u-\check{r}$, jù, let. $t u-r$ 'alli' / esl. aesl. $k \check{u}$, $-k \grave{u}-, i$, rus. to, arus. $t \grave{u}$ 'sí, así' / toc. A kus / B kuse = '¿quién, qué?' (base del tema pron.), A kupre, B kuri/krui, B ya-ka, ya-k, y-käk, t(a)kà 'en efecto' / anat. het. $k u$ / alb. tuk (va con el nom. del participio fem. y refuerza la significación 'mientras, en tanto en cuanto'), $k u$ / celt. airl. toch conj., galés $p y$, airl. $c o, c u$, galés $c w$.

*-ü: gr. $\pi \tilde{u}-\varsigma, \quad \tilde{u}-\varsigma$ / lat. $c \bar{u}-r$, hü-c 'hacia aqui', $\bar{u} s-q u e$ (?) 'seguido (hasta)' / germ. ${ }^{*} h \bar{u},{ }^{*} j u ̈$, an. $\vartheta \dot{u}$ (dat. ¡neutro! del demostr.) / indo-ir. ai. $k \bar{u}$, $t \bar{u}$ 'pero', ir. del SO $k \bar{u} r-\bar{a}$ / bált. let. (dial). jòu / esl. ucr., che. $-k y,-d y$ (en compuestos de base nasalizada) / anat. het. $k u$ (?) / alb. kür ¿¿cuán-

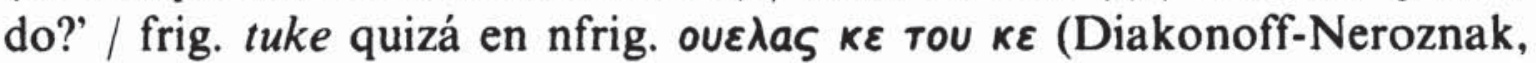
op. cit., pp. 41,136$)$ у то / тоu, partíc. de refuerzo del demostr. $\left(<{ }^{*} t u /\right.$ $\left.{ }^{*} t \bar{u}\right)^{*} t o u$ o $\left.{ }^{*} t \bar{o}\right)$.

Recogida esta serie de finales adverbiales, y de forma previa a la consideración de las circunstancias de su distribución por raíces y lenguas, podemos preguntarnos por la forma más económica de clasificación de todos ellos. Y creo poder reconstruir dos únicos morfemas, cuyas variaciones de silabación en final de palabra dieron lugar al amplio abanico testimoniado. El esquema básico formalizado es PRON (V) $-H_{1}-i$ y PRON (V) $-H_{3}-u$. Siguiendo una teoría laringal estándar:

$$
\begin{aligned}
& { }^{*}-e-H_{1}-i-C>*^{*}-e i\left({ }^{*}-\bar{e}\right),{ }^{*}-e-H_{1}-i-V>*^{*}-\bar{e} i,{ }^{*}-H_{1}-i>*_{-}-i{ }^{*}-i,{ }^{*}-H_{1}-i>*^{*}-a i \\
& { }^{*}-e-H_{3}-u-C>{ }^{*} \text {-ou }\left({ }^{*}-\tilde{o}\right),{ }^{*} \cdot e-H_{3}-u-V>{ }^{*}-o u,{ }^{*}-H_{3}-u>{ }^{*}-u{ }^{*}-\bar{u},{ }^{*}-H_{3}-u> \\
& *-a u
\end{aligned}
$$


4.1.1.1. Varias son las consideraciones que me han conducido a este constructo. La existencia de $-i /-u$ como elemento final de todas las series estudiadas es evidente y no necesita justificación. La otra sonante que se encuentra como formante en muchos casos, $-r$, se superpone a temas pronominales puros: gót. hwar '¿dónde?', palaíta -kuwar (identificador o marca local), lat. quor 'por qué' (todos $\left.<{ }^{*} k^{u} o r\right)$, así como a advs. plenamente caracterizados por el complejo ${ }^{*}-(e) H_{1}-i$ y ${ }^{*}-(e) H_{3}-u$. Por tanto es lícito pensar que no pertenece al esquema más básico de formación adverbial ide. y que ha podido extenderse irregularmente en distintas épocas.

Pues bien, dos sufijos básicos $-i /-u$ (de $-m$ hablo a continuación) bastarían para interpretar la morfología del adv. ide. si no fuera porque alternan con otros advs. que sólo exhiben vocal larga o diptongo largo. Éstos sólo se explican involucrando en su construcción un fonema laringal. Que éste está presente incluso en la base de formas en grado $\emptyset$ que fenotípicamente revelan $\tan$ sólo $-i /-u$ es demostrable ante varios hechos:

a) En el caso de $-i /-u$, sobre todo de $-u$, es muy frecuente un grado $\emptyset$ con vocal larga, explicable por metátesis de una laringal precedente. El punto de vista de los que creen que existió un instr. ${ }^{*} k^{u} u H_{1} /{ }^{*} k^{u} \bar{u}$ choca contra la constatación estadística de que eso significa atribuir un caso precariamente testimoniado en ide. a un tema que es otro tanto, y además exclusivamente adverbial; no explica que no haya un ac. ${ }^{*} k^{\mu} u$ (o) $m$, un loc. ${ }^{*} k^{\mu} u$-ei, etc.

b) En germ., la Brechung de $-i$, optativa y limitada probablemente a casos en que una laringal precede o sigue a ésta ${ }^{8}$, se produce en el

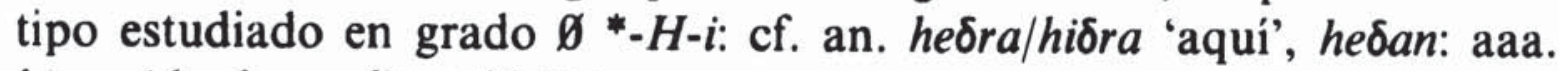
hinan 'desde aquí' $<{ }^{*} k H_{1} i-$.

c) En indo-ir., la existencia junto a $k(u) v$-a/kava-, claramente alargados, de iv- $a$ 'como', que es su paralelo relativo, permite concluir que también ap. $y \bar{a} v-\bar{a}$ 'tanto tiempo como', que convive con ciy $\bar{a}-k a r a m$ 'cómo de grande' < ${ }^{*} k^{\natural} \bar{i}$ y avā 'así(?)' $<{ }^{*} H_{2} e u$, tiene el mismo origen y proviene de un grado $\mathrm{P} * i e H u--\mathrm{V}$.

d) De manera más consistente que el sufijo $-r$, la sigmatización opcional se produce en ide. exclusivamente sobre advs. perfectamente caracterizados como tales, nunca sobre temas pronominales puros, y acarrea en muchos casos el grado $\emptyset$ de aquéllos. Ello implica dos consecuencias inevitables:

- Dado que, en el ejemplo concreto de ${ }^{*} k i$-, que puede recibir una

${ }^{8}$ L. Connolly, «Altnordisch ē < indogermanisch ì, KZ 97, 1984, pp. 267-80. 
- $s$ optativa, parece haber conflicto entre su estatuto adverbial y su existencia en ciertas áreas ide. como tema pronominal, cabe pensar, como ya hizo Pedersen, que el tema pronominal se ha constituido sobre una primitiva partíc. En cuyo caso mantengo que ${ }^{*} k i-,{ }^{*} t i-,{ }^{*} d i$ - comportan una característica adverbial.

- La selección por la que, de los dos posibles grados $\emptyset \mathrm{de}{ }^{*}-H-i / u$, a saber ${ }^{*}-i\left[{ }^{*}-\bar{\imath}\right] /{ }^{*}-a i,{ }^{*}-u\left[{ }^{*}-\bar{u}\right] /{ }^{*}-a u$, sólo ${ }^{*}-i\left[{ }^{*}-\bar{l}\right] /{ }^{*}-u\left[{ }^{*}-\bar{u}\right]$ es sigmatizado

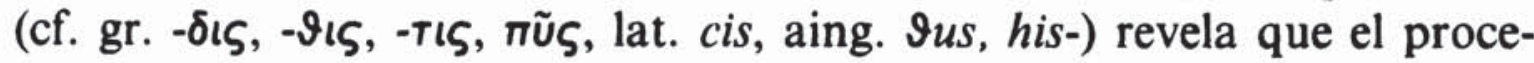
dimiento es lo bastante antiguo como para que el doble segmento sólo pueda aparecer en su grado $\varnothing$ anteconsonántico. Y al revés, esto confirma que había un fonema laringal ante la sonante, puesto que la $-s$, de lo contrario, aparecería arbitrariamente sobre ambos resultados.

e) No hay solución alternativa verosímil a la presencia de un fonema laringal en casos como gr. vaí, kai, o. svai, toc. B nai, etc. No ha existido nunca un loc. fem. en *-ai separado del dat. *-āi.

f) Existen claras alternancias con forma de timbre * $a$ que deben considerarse como variantes más simples de ${ }^{*}-\mathrm{H}-\mathrm{i} / \mathrm{u}$, sea en virtud de una relativa independencia del segmento sonántico frente al laringal, que sería el caracterizador adverbial propiamente dicho, sea porque a veces se sentía secundariamente que la doble caracterización formal no era funcionalmente opositiva. Cf. el sufijo germ. ${ }^{*}$-na frente a $-n \bar{e}$, toc. -m en kospre-m ‘¿cuánto?', frente a A -ne / B -nai, o. dat < *dH-t- 'de', frente a lat. dē, y o. $\sigma_{F} a$, peligno sua 'y' frente a o. svai, lat. si.

g) El final *-ei parece coincidir con la terminación de loc. pronominal. Sin embargo hay que atender a un hecho evidente, aparte de la inexistencia en varias ramas de este caso, y en concreto en la flexión pronominal: las ramas que sí distinguen un loc. pronominal lo forman con una desinencia distinta, y/o sobre un tema diferente. Ejemplo de lo segundo es el o. (cf. sólo exseic < * $e k$-s- de caso oblicuo frente a *ekde casos rectos, eizeic, eisei $<{ }^{*}$ eis de oblicuo frente a ${ }^{*} i$ - de recto), el celtib., donde se conoce un loc. śomei < *so- (frente a advs. como airl. cé $($ cia $)<{ }^{*} k i /{ }^{*} k e i$ : ogám. COI < $\left.{ }^{*} k o i\right)$, y en cierto modo el germ., donde las gramáticas encontramos que an. hvi es un dat. neutro del pron. interr. (!), y que aing. $\vartheta \bar{y}, h w \bar{y}$ son instr. (a pesar de su uso adverbial y conjuncional), aunque estas lenguas forman sus dats. pronominales por medio de *-sm- (gót. hwamma, an. hveim, aing. hwaem). Ejemplo de ambas cosas es la rama bált.: cf. lit. šiamè, jamè $\left(<{ }^{*}\right.$-en), la esl. (cf. aesl. tomĭ, sim̌ < < -en/*-in), el ai. (cf. tásmin, asmin), el av. (cf. ahmi, ahmya, kahmi; el ap. no distingue un loc. pronominal). Con todos estos datos, teniendo en cuenta, por un lado, que estas lenguas conocen advs 
como ${ }^{*} k e i,{ }^{*} t e i,{ }^{*}$ nei y, por otro, que las hay que sólo tienen éstos y ni rastro de un loc., resulta dificil imaginar que esos advs. proceden de paradigmas perdidos. Dado que la flexión del pron. con género se asemeja a la del sustantivo temático, la presencia de los advs. ${ }^{*} n i,{ }^{*} t i$, ${ }^{*} k i$, etc., resulta más bien incómoda. Por ello, reconstruyo en este caso ${ }^{*}-\mathrm{H}_{1}-i$ $-\mathrm{C}$, no *ei.

h) Otro tanto cabe decir de la desinencia de instr. en relación al final adverbial que reconstruyo como ${ }^{*}-\tilde{o}$. Empiezo por reseñar que no es licito contar entre los «antiguos instrs. ide.» formas que aparecen sincrónicamente como dats. en las lenguas; hay autores que creen que existe una antigua terminación de dat. temático ${ }^{*}-\bar{o}$, que pervive $\mathrm{p}$. ej. en lat. y gót., secundariamente caracterizada como ${ }^{*}-\bar{o} i$ por contaminación con la terminación atemática *-ei. A esto se añade el testimonio (dudoso para mí) de determinados grupos adverbiales de función local-direc-

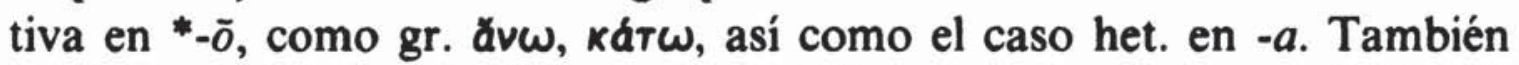
puede que el lat. y el gót. hayan perdido ante consonante su *-i final, a pesar de la temprana regularización de esta desinencia, en virtud de lo innecesario de su doble caracterización ${ }^{*}-\bar{o}-i$ en su oposición formal con el resto del paradigma.

Por lo demás, ésta es la situación del instr. pronominal en las lenguas: en indo-ir., se forma mediante una desinencia especial: así téna, amú-nā, por casificación de antiguas formas adverbiales. (La situación de $y \bar{a}$ o $t \bar{a}$ es ambigua en av. y depende a menudo de la traducción; $A I W$ los traduce siempre como advs.) Similar situación en aesl.: cf. če$m \check{\imath}, \check{c} i$-mĭ. Sólo en bált. aparecen formas como (lit.) juõ, $k u o ̃$ en situación semiparadigmática, que es claramente adverbial en los escasos ejemplos del aprus. En het. existe un tipo en -danta de origen adverbial. El toc. A conoce un instr. en $-y o$ procedente de la aglutinación de un adv. Dado que el resto de lenguas conoce advs. en ${ }^{*}-\bar{o}$ y no un instr. pronominal, y puesto que es claro que gran número de instrs. pronominales proceden de casificación de advs., podemos plantearnos si en el caso del bált. la flexión pronominal se ha dotado de un instr. procedente del adv. en ${ }^{*}-e H_{3}-u$ por rima con el caso nominal en ${ }^{*}-\bar{o}<{ }^{*}-o H_{1}$, sea éste a su vez innovación de ciertas ramas o no. Como el adv. aglutinado de tema *ne- del instr. pron. indo-ir. había sido ya utilizado para la expresión del caso ilativo, esto es tanto más comprensible.

i) En ocasiones, la presencia de la laringal ha alterado los mecanismos fonéticos evolutivos esperables para ciertos fonemas. Creo que éste es el caso de ide. ${ }^{*} k^{*} u$-, que constituye una incógnita por varias razones, entre ellas el hecho de que $-u$ parece no ser incompatible en la mayoria 
de las lenguas con el carácter labiovelar del tema. Se han propuesto diversas explicáciones poco creíbles:

Sturtevant parte, en sus trabajos, del supuesto de que la labiovelar ide. consistía originalmente en dos fonemas distintos indo-het. ${ }^{*} \mathrm{y}^{*} u$. Así, esperamos labiovelar ante vocal en ide., como en el conocido tema ${ }^{*} k^{u}{ }^{u}$ - $<{ }^{*} k w o-$, ante consonante en cambio ${ }^{*} k u$-, como en el tema adverbial ${ }^{*} k u$-, que aparece como ai. $k u$-, gr. $\pi u$-, etc., por analogía de * $k w o$ - Ahora bien, esta teoría no explica, en primer lugar, la función de $-u$ - en el tema del interr. En segundo lugar, no explica la diferencia de tratamiento del segmento ${ }^{*} k u$ - con otros casos ide. de labiovelar ante consonante, donde, como es sabido, labiovelar y velar se neutralizan en general, y sobre todo ante dental, donde nuestro ${ }^{*} k u$ - aparece con más frecuencia. En tercer lugar, sería éste el único caso en que el paso al grado $\emptyset$ de un tema pronominal convierte a éste automáticamente en un adv. o en un tema exclusivamente adverbial. La suposición de que nuestra base desciende del grado $\emptyset^{*} k^{*}$ - de una labiovelar real de la protolengua encuentra el mismo inconveniente. La única posibilidad de explicación de no-neutralización de velar y labiovelar ante consonante sería de carácter morfológico, y se basaría en la necesidad de preservar la identidad de la serie del interr. frente al demostr. ${ }^{*} k e /{ }^{*} k o-$.

Hamp (cf. n. 4) ha sostenido la idea de que el anat. sólo conoce secuencias $/ \mathrm{ku} /=[\mathrm{ku}] /[\mathrm{kw}]$, reinterpretadas como labiovelar unitaria en época ide. post-anat. Frente a ide. ${ }^{*} k^{u} u$-, galés $c w$ representa ide. ${ }^{*} k u$, que se retrotrae al estado de cosas pre-anat. Ahora bien, desde nuestro punto de vista, en el caso de ide. ${ }^{*} k^{u} u$ - uno no ve muy bien de dónde ha salido la $2 .^{a}-u$.

Por otra parte, si supusiéramos que ha habido un tema en grado $\emptyset$ ${ }^{*} k^{*}$-, o ${ }^{*} k u$-, paralelo al demostr. ${ }^{*} i$-, queda en la incógnita no sólo el resultado labiovelar en las lenguas, sino la inexistencia, junto a lat. is, $i d$, gót. is, ita, de un pron. atemático ${ }^{*} k u s /{ }^{*} k u d o^{*} k^{*} s /{ }^{*} k^{u} d$. Tampoco hay restos de un pron. ${ }^{*} k^{u} u$ - frente a ${ }^{*} k^{u} i$-. Hemos visto que, para las formas para las que debe reconstruirse ${ }^{*} k^{*} \bar{u}$, se habla de un instr. ${ }^{*}-H_{1}$. Ahora bien, un instr. en ${ }^{*}-H_{1}$ de ${ }^{*} k^{*}$ - sería ${ }^{*} k^{*} H_{1}>{ }^{*} k^{*} a$; uno de ${ }^{*} k u$-, sería ${ }^{*} k u H_{1}>{ }^{*} k \bar{u}$. Yo creo que ${ }^{*} k^{\mu} u$ - es realmente un grado $\emptyset{ }^{*} k^{*}-H_{3}$ $u$, y que la presencia de laringal ha impedido en muchos casos (en todos, si galés $c w$ es analógico) la deslabialización.

j) El papel de la laringal en la no-asibilación de $-t i<{ }^{*}-t H_{1}-i$ en gr. y zi-anat. no es claro, aunque en gr. una tendencia generada tempranamente puede explicar la no-asibilación tan común de los advs. en - Tl (en

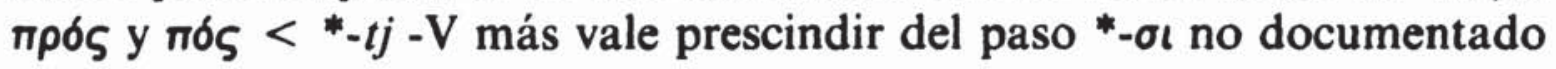

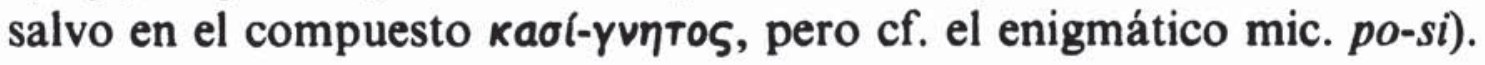


4.1.1.2. La distribución planteada en nuestra hipótesis, según la cual un alargamiento $-u$ va precedido, necesariamente, de ${ }^{*}-H_{3}, \mathrm{e}-i$ sólo de ${ }^{*}-H_{1}$, se deduce de los hechos siguientes:

a) Existen testimonios patentes de diptongo largo, sobre todo en el caso de *-ē-i.

b) En el resultado normal de grado $\mathrm{P}$ anteconsonántico, la solución esperada, respectivamente *-ei y *-ou, es la más común; un grado $\mathrm{P}^{*}$-oi por *-ei, menos frecuente, es fonéticamente normal partiendo de * $o H_{1}-i$, que quizá es producto de la enclisis en etapas primitivas; del resultado *-eu conozco escasos ejemplos (aesl. y rus. - $\check{c} u$, cf. $-k a,-k y$ ) y debe de provenir de un proceso analógico.

c) Con frecuencia, formas satélites de una misma constelación aparecen alargadas por un mismo sufijo. Así, en germ. (an.) tenemos resultados de *iō-r, *iau-r o *iou-r y *iū-r, frente a ${ }^{*} k i-r$, ${ }^{*} k e i-r$ y ${ }^{*} k e \bar{e} i-r \mid$ ${ }^{*} k e \bar{e}$; en plat. poseemos, con toda probabilidad, ${ }^{*} k^{u} \bar{u}-r$ o ${ }^{*} k^{u} O u-r /{ }^{*} k^{u} \bar{o} u-r$ frente a ${ }^{*} k^{u} \bar{o}-r$. Esto induce claramente a la hipótesis de que el alargamiento por medio de $-r$, si bien estructuralmente secundario, es anterior al grado $\emptyset$ y al momento en que aparecen las variantes fonotácticamente condicionadas de ${ }^{*}-e H_{1}-i y^{*}-e H_{3}-u$. Es posible, además, que $-r$ pudiera, alterado por este mismo juego de condiciones, actuar vocálica $o$ consonánticamente.

d) En numerosas ocasiones, la isofuncionalidad de las formas vinculadas es evidente. Así ocurre con los enclíticos y preps. esl. provenientes de ${ }^{*} k^{u} \mathrm{ou} /{ }^{*} k^{*} a u,{ }^{*} k^{4} \bar{u},{ }^{*} k^{*} \bar{o}$, con las conjs. condicionales/concesivas bált. < ${ }^{*} i \bar{e},{ }^{*}{ }^{i} e i,{ }^{*} i e \bar{i} i$ o itál. $<{ }^{*} s(u) e i,{ }^{*} s(u) a i$, con los prefs. ai. en que por fuerza debe reconstruirse ${ }^{*} k^{4} \bar{u},{ }^{*} k^{*} o u$, con los advs. lativos lat. $<{ }^{*} g h \bar{u} /{ }^{*} g h o u,{ }^{*} g h \bar{o}$, con los advs. primitivamente locales gr. < ${ }^{*} k a i$, ${ }^{*} k \bar{e},{ }^{*} k e i$, con los noms. pronominales del germ. occidental, producto de hipóstasis de ${ }^{*} k e \bar{e} i,{ }^{*} k e i,{ }^{*} k i$.

e) A veces, para una raíz, sólo está testimoniado uno de los dos alargamientos, y su aislamiento, además de la sinonimia que muestran en general, evidencia su unidad y la recíproca ligazón de sus términos: y así tenemos tan sólo ide. ${ }^{*} n(e) H_{1}-i$, adv. afirmativo en gr. vaí, ví, veí y en lat. $n \bar{e}, n a e$, sufijo en pgerm. ${ }^{*}-n a i,{ }^{*} n e i,{ }^{*}-n \bar{e}, o^{*} d(e) H_{1}-i$, afirmativo en gr. $\delta a i, \delta \dot{\eta}, \delta \varepsilon i$. Más abajo hablaré de la aparición del segmento * $d h(e) H_{1}-i$ en advs. complejos y de la dudosa existencia de ${ }^{*} d h(e) H_{3}-u$.

f) Ocurre igualmente que, de dos advs. posibles para una raiz, se emplea sólo uno de ellos para entrar en composición o derivación con un segmento preonominal o adverbial de base. $\mathrm{Y}$ así, los temas puros nominales ${ }^{*} \mathrm{H}_{2} \mathrm{eu}$-, ${ }^{*}$ per $_{3}-$, el tema pronominal ${ }^{*} m e-$ y el adv. pronomi- 
nal ${ }^{*} k m$ / ${ }^{*} k o m$ reciben un adv. ${ }^{*}-t e H_{1}-i$, pero nunca ${ }^{*}-t e H_{3}-u$. Véanse los ejemplos menos susceptibles de ser analógicos:

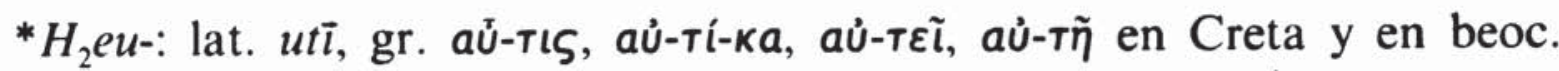

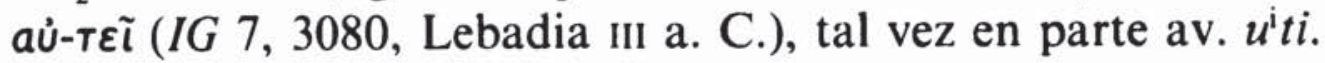

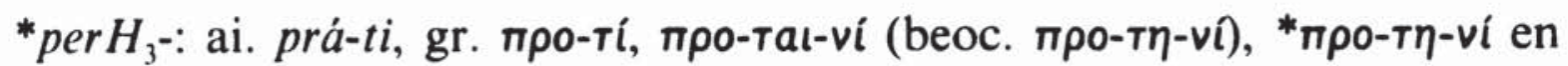
beoc. $\pi \rho 0-\tau \varepsilon l-v i ́\left(B C H 102\right.$, p. $364, \pm 287$ a. C.), panf. $\pi \varepsilon \rho \tau^{\prime}$, o. pert, let. pre-tĩ/-tie, che. pro-ti, aesl. pro-tivŭ (*-tei), het. parza $\left(^{*}-t i\right)$.

${ }^{*} m e-$ gr. $\mu \varepsilon$-тá, $\mu \varepsilon$-тai (?), germ. ${ }^{*} m e-t a,{ }^{*} m e-t i,{ }^{*} m e ́-t i$.

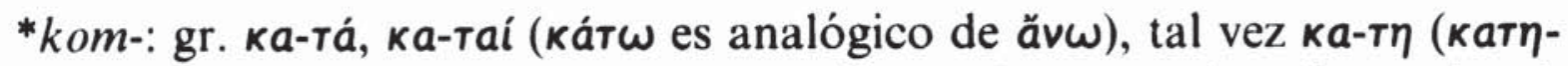

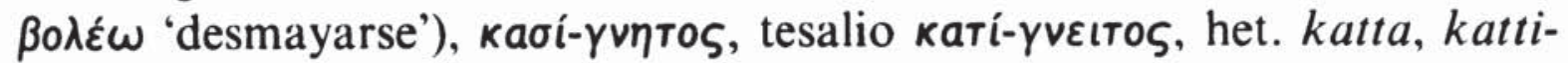
${ }^{*}{ }^{*}-t(e) i$, an. hund- 'muy' , airl. cét-, ceta-/cita- 'con' < pcelt. ${ }^{*} k a n t a$, galés cennym $<{ }^{*}$ kanti-mi ${ }^{10}$.

4.1.2. Finales nasalizados (a diferencia de los tipos anteriores, ofrezco por adelantado formas integradas en advs. complejos para obtener todas las variantes fonéticas posibles).

${ }^{*} k^{u} e-/{ }^{*} k^{u} O-:$ germ. ${ }^{*} k w a n$ '¿cuándo?', aaa. hwant, hwande 'porque' / lat. cum ‘cuándo?', quam 'cuán', quan-dō ‘cuándo?' / bált. lit. kan, kq 'si' / indo-ir. ai. kam (con nú, etc., reforzando al verbo), kám (posp. de dat.), $k a-\left(<{ }^{*} k^{u} m\right)$ (pref. de valoración cuantitativa), av. $k \partial m$ '¿cómo?', aav. kam prep. con dat. de pers. 'a favor de', nav. kamcit 'de cualquier manera' / esl. aesl. kondu, etc., ‘de dónde?’, rus. kudá ‘adónde?’, etc. / anat. pal. -kuanta (adv. encl.).

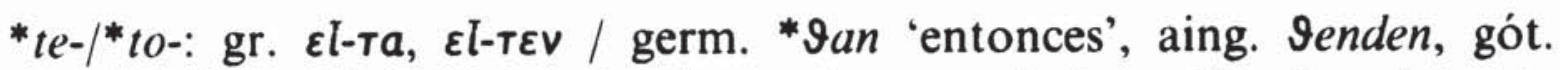
Эande 'mientras' / lat. tam 'tan', tum 'entonces', i-tem 'igualmente', i-ta 'asi', aut 'o', au-tem 'sin embargo' / indo-ir. av. $t \partial m$ 'por eso', u-tá $\left(<{ }^{*}-t m\right)$ ' $\mathrm{y}$ ', iti 'así', it-thā, itthám 'así, iýa/ia $\left(<{ }^{*}-t m\right)$ 'así', pali kattha '¿dónde?', ettha 'aquí' / bált. lit. teñ, tenaĩ, let. tie 'alli', aprus. tēn-ti 'ahora', stwen 'alli', lit. it/yt, ic 'tal como, igualmente', itin 'especial o principalmente' / frig. tan 'alli' o 'entonces'.

${ }^{*} i e-/{ }^{*}$ io-: gr. -i(v) (partíc. de refuerzo) / germ. ${ }^{*}$ jan-di > aing. geond, giend 'hacia alli', gót. jaind / indo-ir. av. yam 'si', yim 'que, para que' / bált. lit. jeñg, jañg 'o, para que, que' / lat. iam 'ya', ex-im, etc. (< ‘im), in-de 'entonces', u. ie-pi 'illic', ie-pru 'inde' $\left(\mathrm{si}<{ }^{*} i m\right) /$ frig.

${ }^{9}$ G. Schmidt, Studien zum germanischen Adverb, Berlín 1962, pp. 277-82.

10 N. Holmer, "A Celtic-Hittite correspondence», Eriu 21, 1969, pp. 23-24. 
ian 'aqui' o 'ahora' / indo-ir. ai. ìm (partíc. anafórica) / celt. celtib. iom (Botorrita) ‘y, también'.

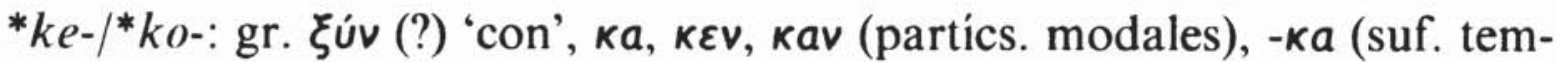
poral) /germ. ga-, ${ }^{*}$ hin-der- $\left(<{ }^{*}\right.$ kem-ter) 'tras', an. handa-n '(desde) más allá' / lat. cum, co- 'con', con-tra / bált. lit. señ 'aquí', aprus. schien 'aquí', schan 'aquí, hacia acá' / esl. aesl. sŭ $(n)\left(<{ }^{*} k m\right)$, se $\left(<{ }^{*} k e m\right)$, son- $\left(<{ }^{*} k o m\right)$ / toc. A š-kam / anat. het. katter 'debajo', -kan (?), het. $k \bar{a}$ 'aqui' / celt. irl. con 'hasta o para que' / alb. $k \ddot{e}-<{ }^{*} k o(m)$-.

*ne-/*no-: lat. num, nam, enim, nem-pe, o. inim, u. enom / esl. aesl. nŭ, rus. no $\left(<{ }^{*}\right.$ nom $)$, aesl. non, s. -cr. antiguo $n u\left(<{ }^{*} n \bar{m} m\right)$ 'pero' / bált. lit. -na, -nq, -ne (suf. aglutinado de ilativo), kanà ‘¿adónde?', nę-sq, nęsang 'pues' / indo-ir. ai. nū-nám 'ahora', nānānám, nā́nā 'de diferentes maneras', vínām 'sin, excepto' (prác. vinu $<{ }^{*}$ vi-nam), -na/-nā (desinencia de instr. pronominal).

*de-/*do-: gr. lat. dum 'mientras', qui-dam 'alguien', i-dem 'el mismo' (o. isi-dum), tandem 'finalmente', quon-dam 'entonces', identidem 'igualmente' $\left(<{ }^{*}\right.$ identident-), dōnique, dōnec, dēnique (?) 'hasta que', ce-do, en-do / germ. 'tō/ *to (prep. directiva) / indo-ir. av. dim (anafórico), ddm, ai. idám 'esto', i-dấn-īm 'ahora', sádam, sáda- 'siempre', kadấm $(R V \mathrm{~V}, 3,9)$, kadá ‘¿cuándo?', prác. cia (*-dm), yádi 'si' / esl. aesl. da 'así, do 'hasta', kondon, por kondu, etc. ‘de dónde?' en el Codex Marianus / bált. lit. da 'hasta', do 'para, a', aprus. din (anafórico), lit. idañt 'para que, como si', lit kadà '¿cuándo?', tadà 'entonces', kadángi 'porque' (let. kad, tad, aprus. kadan, -en), aprus. is-stwen-dan 'desde ahi' (Enquiridión; tal vez falsa lectura por-dau), din (ac. anafórico) / toc. B tot 'hasta tal punto, mientras tanto' (si < ${ }^{*} t o-d \bar{o}^{11}$ ), A $t \bar{a}$ '¿(a)dónde?' (?) / anat. het. -da, -dan-i (dat. pron.), -dan-ta (instr. pron.), todos sobre tema en diptongo.

*dhe-/*dho-: gr. $-\vartheta \varepsilon v,-\vartheta \varepsilon,-\vartheta a$ (suf. abl.), (oủ) $\vartheta \eta v$ (partíc. encl.) / indoir. ai. kú-ha '¿dónde?', ihá 'aquí' (si < *-dhm), pali kaham 'dónde', prác. idham 'aquí', pali kuhim '¿adónde?', ai. bāạhám, nav. bā $\delta a$ 'si', pali ham, ai. hán-ta '¡mira!', ai. kadha-priya- 'por quién amado'.

4.1.2.1. La existencia de una nasal final ${ }^{*}-m$ es evidente y no necesita mayores explicaciones. Se observa, por lo demás, la presencia evidente de una laringal:

"J. Hilmarsson, Studies in Tocharian phonology, morphology and etymology, with special emphasis on the o-vocalism, Reykjavík 1986, p. 49. 
a) De un lado existen grados $\emptyset$ como lat. tam, quam, quizá frig. ian, tan (si no < ${ }^{*}{ }_{i} \bar{e} m$, ${ }^{*} t \bar{e} m$ ), junto a ai. ím, gr. -iv, tal vez av. dim< *dHm, quizá airl. can ‘de dónde?', galés pan 'cuando'.

b) Hay formas con diptongo largo: ai. i-dân-, ka-dấm, aesl. non < *nōm, av. kam, yqum, gr. Inv.

c) Hay una alternancia visible en bloques isofuncionales de formas como lat. num, nam, nem-, o ai. sá-dam, sá-dā, sá-da- / idá, i-dám, i-dān-

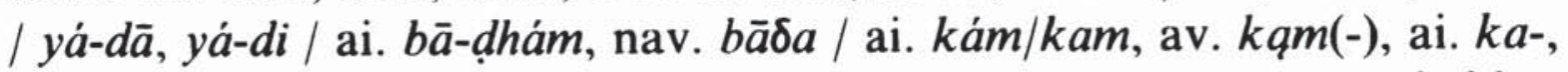
tal vez kam- en kandara- 'barranco', etc. ( $A I G r I I$, p. 85), que hablan contra la hipótesis de finales originarios heterogéneos.

d) En indo-ir. se produce, en casos en que la laringal está en contacto con una dental sorda precedente, aspiración de ésta. Así ocurre en ai. itthám, etc. (itthá con aspiración analógica).

e) Por otra parte, y frente a lo que sucede en el caso de * $-e-H_{1}-i$ y ${ }^{*}-e-H_{3}-u$, las formas con vocal breve procedentes de ${ }^{*}-e m /{ }^{*}-o m /{ }^{*}-m$ sugieren que tanto el elemento laringal como el nasal podian ir en grado P. Es decir que varian, como si el adv. en su totalidad fuera una raíz trilitera dotada de un primer sufijo alternante, entre lo que podriamos llamar un tema I*/-V - H -m/ y un tema II */-H -V -m/, siguiendo pautas tan antiguas como para respetar la ley de equilibrio silábico. Por ello reconstruyo un esquema $\operatorname{PRON}(\mathrm{V})-H(\mathrm{~V})-m$.

f) De la relativa independencia del elemento nasal frente a la categorización laringal hablan alternancias como lat. $i$-ta/i-tem, ai. i-ti/itthám / prác. $i a$, celtib. $u$-ta / av. $u$-i $t i$ / lat. $u$-t (?) // lat. au-tem / ai. u-tá $\left[<{ }^{*}-t(H) m\right]^{12}$.

\subsubsection{El timbre de la laringal es difícil de precisar:}

a) A pesar del sugerente paralelismo que ofrece un sistema $*-H_{1}-i$, *- $H_{3}-u, \quad *-H_{2}-m$, creo que es desechable la reconstrucción por $\mathrm{G}$. Schmidt de un caso modal * $H_{2}-m$, que explica pocos casos, deja casi todos en la incógnita y plantea un problema de convergencia morfosintáctica con los casos ac. (¿e instr.?) del fem. que me resulta de momento insuperable. Además, reconstruye para las formas con vocal breve un sufijo ${ }^{*}-m$ sin laringal ${ }^{13}$. Yo ${ }^{14}$ trato de explicar el caso más claro de

12 Con variantes Hamp, "Why syntax needs phonology", Papers on diachr. synt., 1976, pp. 348-64.

${ }_{13}$ G. Schmidt "Lateinisch tum, tam, quidam und Verwandtes», MSS 47, 1988, pp. 121-31. A. Meillet, "Varia», MSL 15/3, 1908, pp. 193-200, reconstruía *te-, * $k^{\psi} e$ + partícs. como ${ }^{*}$-om, ${ }^{*}$-àm; F. Bader, "Lat. nempe, porceo et les fonctions des particules pronominales", BSL 68, 1973, pp. 27-75, propone ${ }^{*}$ no- / ${ }^{*} k o-,{ }^{*} n a /{ }^{*} k a-$ con nasalización optativa.

14 B. Prósper, La reconstrucción del adverbio indoeuropeo, Madrid (en prensa). 
*-ām, esto es lit. kadà (oriental -u), kadángi, sobre la base de que es la adaptación de un final pbált. *-dam (cf. idañt) a la terminación de instr. fem.; en lit. existen un adv. $k a-d$ - con una multitud aberrante de terminaciones correspondientes a casos de singular y plural lit., por reinterpretación sincrónica de finales adverbiales: cf. kadaĩ ( $\mathrm{adv}$.$) , y sobre ella$ kadaĩs (instr. pl.), kadáise (loc.), nie-kadõs (gen. sg. fem.), etc. Sobre kadà fue alargado el ilativo kanà.

b) En cierto número de casos, la vocal que precede o sigue a la laringal es una *-o-. Sin embargo, existen ejemplos indubitables que exigen -e-. Por ello, la solución más probable es ${ }^{*}-H_{1^{-}}$, que no afecta al timbre de la vocal. Existen ejemplos en indo-ir. de aspiración de sorda o sonora por contacto con ${ }^{*} H_{1}$; esto excluye posiblemente ${ }^{*} H_{3}$ en el caso del subsistema ${ }^{*} t-(e) H(e)-m>$ indo-ir. ${ }^{*}-t h-$, y confirma la reconstrucción de laringal en variantes en que ésta aparecía entre consonante y vocal y es, por lo demás, indetectable en la exploración fonética.

c) Esta constatación ilumina el principio de solidaridad distribucional, que enuncio así: Si una raíz se ha visto alargada en ide. sólo por dos de los tres sufijos mencionados, y en el supuesto de que uno de los dos sea $*_{-}(e) H_{1}(e)-m$, el otro será necesariamente ${ }_{-}^{*}(e) H_{1}-i$ y no $*_{-}(e) H_{3}-u$. Así, tenemos ${ }^{*} n-(e) H_{1}(e)-m /{ }^{*} n-(e) H_{1}-i$ y ${ }^{*} k(e) H_{1}(e)-m /{ }^{*} k-(e) H_{1}-i$. En la mayoría de las familias no hay nada susceptible de interpretarse como un adv. ${ }^{*} k(e) H_{3}-u$. En este caso, los escasos restos posibles de ${ }^{*} k \bar{o}$ (anat., tal vez toc.; en ambos casos es posible ${ }^{*} g h \bar{o}$ ) pertenecen al subsistema ${ }^{*}-(e) H_{1}(e)-m$ y quizá confirman que éste tenía ${ }^{*}-H$ en la raíz *ke-/*ko-, cosa no evidente a partir de los demás ejemplos.

Por lo demás, adelanto que este principio se observa igualmente en los advs. complejos, donde encontramos advs. nucleares como ${ }^{*} i-t(e) H_{1}-i$ e ${ }^{*} i-t(e) H_{1}(e) m$, pero no ${ }^{*} i-t(e) H_{3}-u$, restricción secundaria a pesar de la existencia de un adv. básico ${ }^{*} t(e) H_{3}-u$. Esto se aprecia con más claridad en la sufijación de temas nominales, donde, sea cual sea la base, no existe un sufijo ${ }^{*} t(e) H_{3}-u$.

\subsection{Formación de palabras}

Como se desprende de lo dicho, la variante más simple del adv. pronominal ide. está constituida por adición de un elemento propiamente adverbial, complejo e integrado por dos elementos consonánticos, a una base pronominal simple, como ${ }^{*} k^{\mu} e-/{ }^{*} k^{\mu} o-,{ }^{*} t e-/{ }^{*} t o-$, etc.

a) El fenómeno en sí debe tipificarse como simple adherencia de 
un morfema de carácter flexional. Es decir, el alargamiento cumple una función cuasi-desinencial. Lo llamaré PRON + FLEX.

b) El proceso es anterior a la aparición del grado $\emptyset$. Nada indica que se haya producido con posterioridad a la creación de los primeros casos de la flexión pronominal; en ocasiones, parte de los elementos resultantes han sido clasificados idiomáticamente, debido en parte a la coincidencia de algunas variantes con casos del sistema nominal. Que el mecanismo no debe interpretarse a la inversa se deduce de los hechos reseñados en 2 y en 4.1.1.1 g) h). Además, como he dicho (4.1.1.1 f), en ocasiones encontramos formas que con seguridad poseen laringal, pero no ${ }^{*}-i{ }^{*}-u$ o ${ }^{*}-m$. Podemos pensar que el fenómeno del alargamiento consta estructuralmente de dos fases, sin que esto presuponga cronológicamente dos oleadas; así, un subsistema *- $\mathrm{H}-\mathrm{i}$ puede haber tenido una variante igualmente adverbial *- $H$ desde el principio.

\subsection{Morfosintaxis}

4.3.1. Todo adv. en la protolengua debe contener un elemento cuasiflexivo que permite al lexema al que se adhiere funcionar como tal de manera independiente como predicado de frase, y según las lenguas, secundariamente, como preverbio propio y como prep. o conj.

a) Esto no significa que un elemento puro de carácter pronominal no pueda encontrarse como forma independiente. En primer lugar, hay que distinguir entre morfemas léxicos pronominales de carácter deíctico y de carácter sintáctico. Así, mientras que *de-/*do-, *dhe-/*dho-, *ne-/ ${ }^{*} n o$ - y ${ }^{*} k e-/{ }^{*} k o$ - y probablemente ${ }^{*} s(u) e-/{ }^{*} s(u) o$ - (que exige tal vez un estudio aparte) poseen un contenido eminentemente deíctico, ${ }^{*} k^{u} e-/$ ${ }^{*} k^{u} o-,{ }^{*} i e-/{ }^{*} i o-$ y quizá ${ }^{*} u e-/{ }^{*}$ uo- carecen de otro contenido que no sea el merámente sintáctico. ${ }^{*} t e /{ }^{*}$ to- parece ocupar una situación intermedia; posiblemente en su origen denotaba la mera "demostratividad» por oposición al carácter rel. e interr., como término de neutralización de los diferentes tipos demostr.; sin embargo, al resultar este tema marcado morfosintácticamente como forma pronominal o adverbial, y en tanto se opone en cada lengua a los demás subsistemas demostr., señala positivamente uno de los puntos del campo deíctico o del discurso. A esto hay que añadir la negación, que ocupa un lugar especial y que no trato aquí.

b) La repercusión sintáctica de esta constatación es inmediata. Los temas deícticos no aparecen aislados, al menos en situación tónica o proclítica, porque portadores de nociones deícticas concretas no pueden 
ocupar sin más lugar sintáctico alguno. Cuando éste parece ser el caso, y las condiciones fonéticas impiden interpretarlo de otra manera, puede hablarse, y se habla, aun sin tener en cuenta este principio explícitamente, de abreviación: cf. p. ej. gr. $\delta \dot{\varepsilon}$, que sin embargo, como aparece en segundo lugar de frase, debe haber sido originalmente un enclítico. En todo caso no hay correspondencias en las lenguas que permitan retrotraer el estatuto adverbial de formas puras de este tipo a la protolengua.

En cambio los temas sintácticos aparecen como conjs.; tal vez en la conj. copulativa ide. *ịo- en gót. ja(- $h)$, aunque ésta es en los casos más claros enclítica: así het. -ya, quizá mic. to-so-jo: to-so-de, galo dugiiontiio 'que honran'. Quizás en la conj. disyuntiva *ue, si es distinta del tema de alejamiento nominal ${ }^{*} \mathrm{H}_{2} e u$-, y quizás en casos como het. $t a$ 'y' cuando va sin pron. encl., salvo que éste sea un rasgo ya de una lengua aislada, o que en este uso particular haya que reconstruir ${ }^{*}-\bar{o}$.

c) Suponiendo que sea de herencia ide., el tipo sintáctico anat. de las partícs. iniciadoras de frase no entra en contradicción con la idea antedicha, por cuanto éstas no aparecen como tales, sino seguidas de un pron. enclítico. A diferencia de los enclíticos que voy a tratar a continuación, este pron. encl. no constituye una adición vacía sintácticamente, sino que es vital para el funcionamiento de la raíz pronominal precedente, que queda convertida en tema pronominal soporte. Así ocurre en luv. $a$-, het. $t a$. Het. $s u$, het. pal. $n u$, que pueden llevar adherido al pron. encl., pero también otras partícs. como en het. napa $<{ }^{*} n u$ $a p a$, nasta $<{ }^{*}$ nu-asta, no son estructuralmente elementos radicales puros, a pesar de ser invariables e inanalizables ya, y por ello estos casos citados corresponden al esquema formal que llamo [PRON + FLEX] + ENCL. El tipo het. nakku, takku, nassu (nasma < *nu-asma), quizá airl. nó, galés neu, corresponden a un tipo diferente que estudio en 5.2.3.

d) Cualquier elemento pronominal básico puede funcionar como enclítico de una forma morfosintácticamente caracterizada, sea ésta flexiva (pronominal) o cuasiflexiva (adv.). Es decir, que segmentos mínimos dotados de una noción deíctica o sintáctica la incorporan a otro que, en sí, puede prescindir de ella, dotándolo de un matiz u orientación referencial secundario.

- Un ejemplo que implica elemento sintáctico enclítico es ${ }^{*} k^{y} e$, que, aparte de su función conectiva, se añade, según el antedicho esquema (PRON + FLEX) + ENCL:

A advs. como lat. absque, dōnique -cunque. El proceso se repite para raíces concretas desde antiguo: ${ }^{*} H_{1}(e) t(i)-k^{u} e /{ }^{*} H_{1}(e) t-s-k^{u} e$ (ai. áccha, gr. 


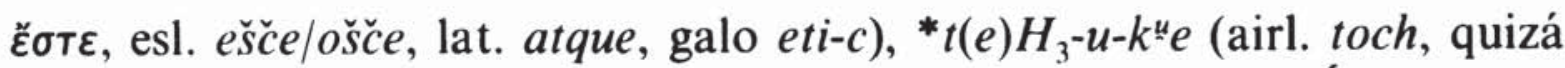
nfrig. Tou $\kappa \varepsilon$, aing. $\vartheta \bar{e} a h$, alb. $t u k),{ }^{*} H_{2} u(-t)-(s)-k^{u} e$ (ai. uccá, av. usca, quizá lat. ūsque, parcialmente gr. aú-te, gót. $-u h$, celtib. $\left.a u k u^{15}\right)$.

A prons. (lat. quisque, lic. tike, gr. öøre). No es fácil discernir la pertenencia de ambos procedimientos a dos etapas distintas, identificarlos, o hacer derivar con claridad uno de otro.

- Un caso que involucra claramente un deíctico es el del enclítico direccional de base ${ }^{*} d e-/{ }^{*} d o-;$ en gr. $-\delta \varepsilon$ se une, como he previsto, a un ac. formado. Existe, en cambio, una amplia isoglosa germ., bált., esl. y celt. de uso preposicional secundario de este tema, sin que sea fácil admitir abreviación proclítica en todas las lenguas. Cf. nuestra explicación en 4.4 a). Sin embargo, hay correlatos isofuncionales con vocal larga *dō en germ., alit. y probablemente celt. y gr.

e) La necesidad de caracterización de elementos adverbiales alcanza a fortiori a los preverbiales y prefijales; por ello, no puede considerarse válida la idea extendida de la prefijación por medio de ${ }^{*} k e, p$. ej. en lat. ceu < ${ }^{*} k e$-iue (Watkins), que en todos los casos tiene alternativas fonéticas y morfológicas, y además en ocasiones, de ser aceptada, admite otras interpretaciones desde el punto de vista de la formación de palabras. En general, la prefijación o preverbación es un fenómeno reservado a los advs. de origen nominal. En las ocasiones comparativamente escasas en que aparece un pref. de origen pronominal, está caracterizado por un elemento categorizador, o bien acompaña, en la misma lengua y función, a una serie de variantes de un elemento que lo estaba: se deduce que el elemento puro se ha adherido idiomáticamente a la serie, desde la posición enclítica, por atracción temática, cuando no resulta fonéticamente de un cruce analógico o corrupción de formas más complejas: en lat. co- (por com-) podemos tener simplemente la constatación de que en final -om representaba una vocal nasalizada. Por ello, diré que el esquema **PREF + ADV no existe.

4.3.2. El procesn de desintegración del sistema adverbial ide. sigue a nuestro juicio varias fases sucesivas, que plantean problemas a medio camino entre la constatación morfonológica y su repercusión morfosintáctica:

a) En fecha anterior a la desaparición de las laringales, el elemento caracterizador ${ }^{*}-H_{1}-i,{ }^{*}-H_{3}-u,{ }^{*}-H-m$ era reconocible como distinto del tema pronominal soporte, y sus posibles oscilaciones articulatorias con-

is $\mathrm{Si}$ es un adv., lo que es dudoso, fonéticamente contaminado con el esquema tratado en 5.2.3. 
dicionadas no alteraban su función básica; es decir, que las variantes fonotácticas y de silabación no descomponen la unidad morfológica.

b) En el momento en que las laringales desaparecen, surge en el seno de la palabra una serie de variantes fonéticas que, a diferencia de la situación anterior, poseen ya carácter fonológico, es decir $*_{-}-i,{ }^{*}-a i$, ${ }^{*}-e i,{ }^{*}-\bar{e} i,{ }^{*}-\bar{e}$. Este hecho carece aún de relevancia en el plano morfológico, por cuanto las formas resultantes son aún satélites de la misma órbita. Sin embargo, la desaparición del condicionamiento articulatorio que regía la variación interna en la fase a) permite ya la polarización de funciones propias de la fase c) (infra). Por lo demás, no hay ya percepción de la distinción sintagmática entre la base léxica, que mantiene asociado al conjunto en la conciencia del hablante, y el morfema flexivo, de manera que es en todos los casos una forma inanalizable la portadora de contenido déctico y contenido sintáctico. Ésta es, desde estadios muy anteriores, la situación de proto-ide. ${ }^{*} n u$, y también la de esp. aqui, si, asi, y sus protoformas lat.

c) Con el paso del tiempo y la dialectalización progresiva, la existencia de formas sinónimas en grandes cantidades se vuelve inútil y surge la confusión en los casos integrantes de sistemas paradigmáticos. Por ello, nuestras variantes

- pasan a aumentar desigualmente el volumen de estos sistemas, siendo analizados otra vez como lexema y terminación, que es remorfologizada y reespecializada;

- se pierden cuando su secuencia fónica no concuerda con los usos más normales en una lengua;

- son depuradas según el dialecto en un proceso mal descrito de selección;

- son seleccionadas e inician a su vez, por su comparativa rareza, un proceso de selección analógica sobre otras formas, con un resultado de amplitud dialectal generalmente limitada.

\subsection{Semántica}

Es dificil discernir el valor original de cada uno de los tres sufijos tratados, porque interfiere el de la raíz a la que se añade, por la dispersión centrífuga progresiva de sus variantes integrantes, y por atracción analógica entre las diversas raíces. Hay que contar con la modificación originada por ulteriores sufijos, como ${ }^{*}-r$. Por fin, es normal que una lengua haya escogido y reespecializado sólo uno de dos subsistemas al- 
ternativos para la misma raíz, lo que dificulta el esclarecimiento de su valor.

a) Sabemos, por un lado, que es improbable que estos elementos hayan añadido una especificación direccional; el carácter loc., abl. o directivo se obtiene de la superposición de varios elementos, de los que trataré más abajo, o bien es producto de la polarización funcional intralingüistica de las variantes adquiridas en direcciones opuestas.

En el tipo ${ }^{*} d e-/^{*} d o$ - se suele admitir un valor direccional original, sobre todo en referencia a preps. y posps. remitibles a ${ }^{*} d \bar{o}$ (para mí a su vez $\left.<^{*} d \bar{o} m\right)^{16}$. La idea de un caso nominal en ${ }^{*}-\bar{o}$, conservado sobre todo en advs., precisa la teoría del sincretismo. Ahora bien: una prep. que indica dirección no porta a su vez normalmente la terminación del adv. o caso direccional, salvo que partamos de una desemantización por la que *do 'hacia alli' da 'hacia'. Además, suele olvidarse que los temas pronominales deícticos se limitan a señalar y no expresan ninguna clase de movimiento.

Creo que el valor direccional de ${ }^{*} d e-/{ }^{*} d o$ - resulta en última instancia de un empleo de referencia local anafórica de esta raíz. Como adv. plenamente formado funciona en av. y aprus. como pron. encl. anafórico, si admitimos que aprus. $\operatorname{din}\left(<^{*} d H m\right)$ y av. $\operatorname{dim}\left(<^{*} d H \boldsymbol{H m}\right)$ son en última instancia advs. = 'ahi' reinterpretados como acs. sin distinción de género. Está también como identificador anafórico en lat. idem, como demostr. (quizá primitivamente anafórico, cf. Brugmann 1904, p. 37) en ai. idám, e indef. en lat. quidam. Podemos imaginar la evolución nocional asi: junto a advs. aislados, formas reinterpretadas como pronominales, y advs. aglutinados como meros sufijos, existen formas puras adheridas como enclíticos a palabras completamente formadas: es el caso de

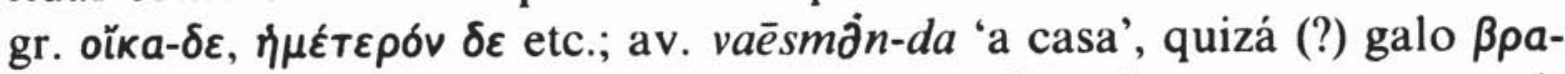
Tou- $\delta \varepsilon$ 'e judicio'. Otras llevan, pospuestos, advs. plenamente caracteri-

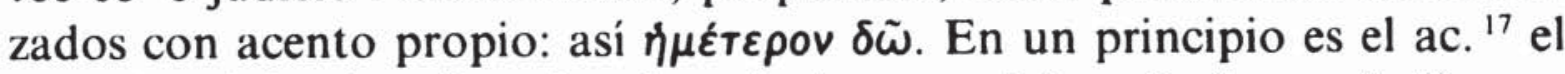
portador del valor direccional, y el elemento $* d$ - se limita a añadir una referencia local anafórica: 'a la casa: ahí', con $\delta \tilde{\omega}$; 'a la casa (+ referencia local intraducible)' con $\delta \varepsilon$. (Quizá es innecesaria la distinción entre si, en última instancia, ${ }^{*}$-de, etc., hacía referencia local a una mención del sust. o pron. en la frase anterior ['éste-el de antes', etc.] o, como digo, implica una mención local a la forma a la que se añade). A conti-

${ }^{16}$ G. Dunkel, «IE conjunctions: pleonasm, Ablaut, suppletion», $K Z$ 96, 1982-83, pp. 178-199; E. Risch, "Die verschiedenen Partikeln $\delta \varepsilon$ im Griechischen», Studi Pisani II, 1969, pp. 831-43.

17 En galo no es claro el caso; si $-\delta \varepsilon<{ }^{*} d \bar{e}$, es una posp. = lat. dēe, airl. di. 
nuación, sea sólo por un uso creciente de la prep., o porque antes ha habido una etapa ide. posposicional, deja de comprenderse este tipo de construcción de sintagmas locales. ${ }^{*}$ de- pasa a entenderse como direccional en relación directa con la pérdida del ac. de dirección; y en su forma pura y en la caracterizada, pasa al uso preposicional en una isoglosa celt.-balt.-esl.-germ., quizá itál. Las correspondencias invitan a ver aquí un adv. ${ }^{*} d(e) H(e) m$; sin embargo, gót. $d u$ (y en parte quizá airl. do prep., to- prev.) podría proceder de ${ }^{*} d H_{3} u$, y por ello es posible que parte de esta gran zona haya desarrollado ${ }^{*} d(e) H_{3}-u$, como bált.-esl. ${ }^{*} d h(e) H_{3}-u$. No es claro si celt., itál. *dē 'de' proceden de este subsistema o de ${ }^{*} d(e) H_{1}-i$, como gr. $\delta \eta ́, \delta a i ́, \delta \varepsilon \tilde{i}-v a$, pero en este último caso, su paso a prep. se habrá visto influido por ${ }^{*} d(e) H(e) m$.

b) Estos elementos sólo añadían un valor nocional de índole local o modal; la identificación de este último plantea problemas cuando se adhiere a un tema con carga local y no puramente sintáctica, como *de$/^{*}$ do-, ${ }^{*}$ dhe- $/{ }^{*}$ dho- o ${ }^{*} k e-/{ }^{*} k o-$. El valor temporal puede surgir de cualquiera de los tipos; cf. en cuanto al origen modal al. wie en la acepción 'cuándo' o el doble valor lat. de cum. Asimismo el valor abl. puede ser originalmente uno causal y el directivo uno final, ambos a su vez modales en un principio.

c) En ocasiones parece vislumbrarse, dentro de la inconstancia de los datos, un valor local para ${ }^{*}-H_{1}-i$ y uno modal para ${ }^{*}-H_{3}-u,{ }^{*}-H-m$. En general, se observa mayoritariamente que los advs. con raíz ${ }^{*} d e-/$ *do- $\mathrm{y}^{*}$ dhe- $/ *$ dho- determinan con claridad el valor local independientemente del sufijo, como se verá sobre todo en el apartado de advs. complejos; por lo demás, *- $\mathrm{H}-\mathrm{m}$ es generalmente modal; en ${ }^{*}-\mathrm{H}_{3}-u$ frente a *- $H_{1}-i$ se inclina ligeramente la balanza a favor del uso modal, pero es muy posible que originariamente ambos sufijos, conocidos en el tipo nominal, hayan sido locales por oposición a un *-H-m modal exclusivamente de ámbito pronominal.

\section{AdVERBios PRONOMINALES COMPLEJOS}

\subsection{Morfología}

Estos advs. son fundamentalmente el resultado de combinaciones de los subsistemas descritos en 4 entre sí y con bases nominales y pronominales diversas. 


\subsection{Formación de palabras}

Como vamos a ver, los mecanismos de formación, si bien obedecen a pautas claras, revelan diversos tipos de relación sintagmática entre sus componentes.

5.2.1. Destaco, en primer lugar, el proceso de sufijación.

a) Como hemos visto en el apartado dedicado a los advs. pronominales básicos, y como sucede en general en el sistema nominal y pronominal ide., el sufijo adverbial tiene final consonántico, o sea distinto de -e/-o. Quiero decir con ello que debe ofrecerse una explicación alternativa (cf. infra) a los casos de ai. iva, retrotraído tradicionalmente a ${ }^{*} i$-ue, y gr. $\left\lfloor\delta \varepsilon\right.$, aparentemente $<{ }^{*} i$-de. ${ }^{*} i$ no existe como partíc., o conector independiente, por lo que no puede hablarse de yuxtaposición de elementos simples. Por otro lado, ue o* de, como se desprende de 4.3.1 d), puede funcionar como enclítico, pero no sobre un elemento puro no previamente caracterizado por un morfema flexivo. El esquema ${ }^{* *}$ PRON + ENCL no existe. La reconstrucción lat. ceu $<{ }^{*} k e-i-u e$ entendida como *kei-ue es correcta según [PRON + FLEX] + ENCL; pero entendida como ${ }^{*} k e$-iue ${ }^{18}$ vulnera simultáneamente los principios «no **PREF + ADV» y «no **PRON + ENCL». En ai. iva opto (cf. 4.1.1.1 c) por una resufijación indo-ir. de PRON + FLEX que abre la puerta a interesantes paralelismos formacionales; y el ejemplo gr. $1 \delta \varepsilon$ probablemente es cipriotismo por $\eta \dot{\eta} \delta \varepsilon^{19}$, yuxtaposición puramente gr.

b) Por tanto, el primer tipo de advs. complejos es el integrado por un tema pronominal soporte sufijado por medio de un adv. perfectamente formado, en el sentido de que a su vez se descompone en base pronominal y morfema flexivo. El esquema será PRON + [PRON + FLEX].

c) Lo que distingue morfológicamente este tipo de PRON + FLEX es precisamente que este último constituye el segundo elemento del anterior. Esto es, desde el punto de vista estructural y quizá cronológico, PRON + FLEX es un adv. independiente que se incorpora como morfema flexional a una base pronominal. De ello se sigue lógicamente que, si el proceso de sufijación del tipo básico PRON + FLEX surge de la adaptación y extensión de un morfema flexivo de origen tal vez nominal, el de PRON + [PRON + FLEX] constituye una

\footnotetext{
18 C. Watkins, «Etyma Enniana», HStClPh 77, pp. 195-206.

19 P. Wathelet, "Mycénien et grec d'Homère: 2. La particule kaí», $A C$ 33, 1964, pp. $10-44$.
} 
aglutinación propiamente dicha, idéntica a la del caso *-bhi en el sistema nominal frente $a^{*}-e i o^{*}-(e / o)-s$. Esto implica además que no se trata de un derivado, ya que ni formacional ni funcionalmente puede dividirse el adv. en un tema flexional consistente en raiz y formantes o sufijos y una terminación: en resumen, hablar de un derivado implica un análisis incorrecto [PRON + PRON] + FLEX, más específicamente $[$ PRON + SUF] + FLEX, que diacrónicamente y en este apartado no tiene sentido.

d) Podemos incluir aquí:

- El tipo local *i-d(e) $H_{1}(e) m$ (ai. i-dấ/i-dám, i-dān-, lat. idem, o. isídum), y quizá ${ }^{*} i-d h(e) H_{1}(e) m$.

- El tipo modal ${ }^{*} i-t(e) H_{1}-i /{ }^{*} i-t(e) H_{1}(e) m$ en lat. ita/item, ai. itthám/ $i t i$, pali ettha, av. $i \vartheta a /-\bar{a}$, lit. it/itin/ic, gr. $\varepsilon l \tau a / \varepsilon l \tau \varepsilon v$, galés $y t$.

- El tipo local $*^{*}-d h(e) H_{1}-i$ en lat. $i b \bar{i}$, gr. $\mid 9 a t-$ impvo. ai. y gr. ${ }^{*} i$-dhi, y también het. ked, kedi, y luv. jer. ìtilïtaī, dats. sg. y pl. de i'éste': cf. infra $5.2 .2 \mathrm{~g}$ ).

${ }^{*} i$ - en estos elementos primigenios puede por su extensión haber sido el generador etimológico de ${ }^{*} k^{u} o-d(e) H_{1}(e) m,{ }^{*} t o-d(e) H_{1}(e) m$.

Por otra parte, existe un adv. ${ }^{*} k^{\mu}-o-n(e) H_{1}-i /{ }^{*} k^{\mu} o-n(e) H_{1}(e) m$ del que conocemos sobre todo la forma interr., correspondiente a diferentes demostr.: cf. germ. ${ }^{*} k^{u} o-n a,{ }^{*} k^{u} O-n \bar{e}$ (quizá ${ }^{*} k^{u} o-n i$ en an. hvén-aer), nav. $k a n a$, ap. yanaiy, airl. cuin $<{ }^{*} k^{u} o-n i$ (galo poni), lit. kanà, het. demostr. kani $^{20}$.

e) Aquí es preciso plantear un problema. Según la lengua, la raíz del interr. que corresponde al demostr. ${ }^{*} i$ - es ${ }^{*} k^{u} o$ - o ${ }^{*} k^{\mu} u$ - es un grado $\emptyset{ }^{*} k^{u} H_{3}-u$ del adv. básico ${ }^{*} k^{u}(e) H_{3}-u$. Por lo demás, hay casos en esl., lat. y ai. que avalan la existencia de ${ }^{*} k^{u} H_{3} u$ - $d h(e) H_{1}-i$ : así lat. $u b i / u b i \vec{i}, u$. pufe, aesl. kŭdi (Psalt. Sinait. 78, 10), ai. kúhayā (cf. AIGr III, p. 564), pali kuhi-m '¿dónde?'. Pero está representado en varias lenguas ${ }^{*} k^{u} H_{3} u$ dhe (aesl. küde, ai. kúha, si no < *-dhm, final inseguro en lid. kud, galés $k u\left(t^{\prime}\right)$. Ante esto cabe pensar:

- Que este último pertenece al tipo ADV + ENCL, y que sólo por relación secundaria con el demostr. ha adoptado el sufijo ${ }^{*}-d h(e) H_{1}-i$. Del mismo modo se aclara por qué hay gr. $\pi \delta-\vartheta \varepsilon v$ y $\pi \rho \delta \sigma-\vartheta \varepsilon / \pi \rho \delta \sigma-\vartheta \varepsilon v$, pero no ${ }^{* *} \pi \delta-\vartheta \varepsilon$. Mientras que $\pi \delta-\vartheta \varepsilon v$ corresponde al presente apartado y $\pi \rho \delta \sigma-\vartheta \varepsilon$ al de ADV + ENCL, $\pi \rho \delta \sigma-\vartheta \varepsilon v$ es un tipo mixto hipercaracterizado y expandido, $\mathrm{y}^{* *} \pi \delta^{*}-\varepsilon \varepsilon$ no existe, o de lo contrario hubiera

${ }^{20}$ Es inseguro un adv. ${ }^{*} i-n(e) H_{1}-i,{ }^{*} i-n(e) H(e) m$, construido como demostr. equivalente a ${ }^{*} k^{\sharp} O-n-$; habría pervivido en iva con aspiración secundaria, y tal vez en $u$. inom, o. inim. 
transgredido la regla «no **PRON + ENCL». No es una coincidencia desde el punto de vista formacional que los tipos gr. пó- $\tau \varepsilon$ y $\pi \delta ́-\sigma \varepsilon$ (suponiendo que no procedan de $\left.{ }^{*}-t H_{1}\right)$ carezcan completamente de paralelos etimológicos.

- Que tempranamente se ha producido una hipóstasis del adv. ${ }^{*} k^{u}(e) H_{3}-u$, que se ha constituido como tema pronominal, recibiendo en consecuencia el sufijo ${ }^{*}$-dh(e) $H_{1}-i$ y pasando al grado $\emptyset ;{ }^{*} k^{\sharp} H_{3} u$-dhe es un adv. independiente de formación anterior a este estado de cosas, o bien ${ }^{*}$-dhe fue atraído analógicamente a sufijar lo que ya era el equivalente de un tema pronominal. El tema ${ }^{*} k^{4} \mathrm{H}_{3} u$ - sirve como base igualmente a ${ }^{*}$-tos y ${ }^{*}$-ter y no muestra alternancia visible.

El demostr. ${ }^{*} i$-dhe es analógico en esl., y no debe verse en indo-ir. si, como creo, hay que restituir un tema ${ }^{*} i-d h(e) H_{1}(e) m ;{ }^{*}-d h(e) H_{1}(e) m$ no se distingue de ${ }^{*}-d(e) H_{1}(e) m$ en muchas lenguas y pudiera ser una innovación indo-ir.-gr. $\mathrm{Y}^{*}-d h(e) H_{1}(e) m$, cuya variante ${ }^{*}-d h(H) m$ no se distingue en indo-ir. de ${ }^{*}$-dhe, puede haber tenido un correlato interr.

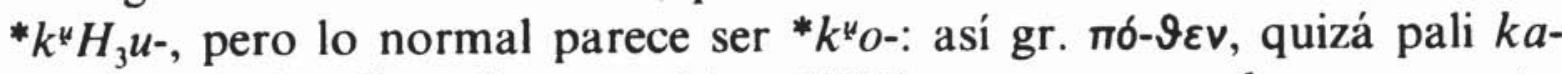
ham, y ai. ka-dha- si es $={ }^{*} k^{*} o-d h(H) m$ y no un adv. compuesto ${ }^{*} k^{u}(H) o m-d h(H) m$ o un adv. + encl. ${ }^{*} k^{\mu}(H) e / o m-d h e$.

En el caso del demostr. modal ${ }^{*} i-t(e) H_{1}-i /{ }^{*} i-t(e) H_{1}(e) m$ no hay correlato interr. seguro sufijado por ${ }^{*}-t(e) H_{1}-i$, salvo si se juzga como tal el representado por los cuantitativos ai. káti, lat. quot, luv. kuwatti 'como, donde, cómo'. El mismo demostr. ${ }^{*} i-t(e) H_{1}-i$ aparece escasamente representado (tal vez en parte ai. it $i$; lit. ic). Para ${ }^{*} i-t(e) H_{1}(e) m$ conservamos un interr. ${ }^{*} k^{u} o-t(e) H_{1}(e) m /{ }^{*} k^{u} o$ - $t H$ en het. kuwat 'por qué', kuwatta(n) 'adónde', ai. kátham/káthā 'cómo', y ${ }^{*} k^{u}\left(H_{3}\right) u-t(e) H_{1}(e) m /{ }^{*} k^{u}\left(H_{3}\right) u-t H$ (ai. kútha/av. $k u \vartheta a$ ), tal ver surgido de contaminación con ${ }^{*} k^{\mu}\left(H_{3}\right) u-d h$-.

f) No es fácil decidir si se debe encuadrar en el capítulo de los derivados o en el de los compuestos el tipo caracterizado por un tema nominal y un adv. simple, del tipo ${ }^{*}$ per $H_{3}-t(e) H_{1}-i,{ }^{*} H_{2} e u-t(e) H_{1}-i$ / $-t(e) H_{1}(e) m,{ }^{*} H_{l} e n-d(e) H_{1}(e) m$, (het. anda[n], gr. ह̌voov, lat. endo y endō en la inscr. de Duenos ${ }^{21}$, alb. ndë y airl. ind dudosos) formalizable como NOM + [PRON + FLEX]. Dado que un tema nominal de por sí y en cualquiera de sus formas puede comportarse adverbialmente sin recibir una caracterización como la de los advs. pronominales (no existe un «loc. Ø» de una raíz pronominal), su estatuto dentro de este tipo es ambiguo y ambas soluciones son probables. En todo caso hay que integrar en el conjunto ${ }^{*} n \check{u}-n(e) H_{1}-i$ (aesl. nynĕ, nŭně, lit. nunaĩ, de no ser

${ }^{21}$ H. Eichner, «Reklameiamben aus Roms Königszeit», Sprache 34, 1990, pp. $212,229$. 
analógicos; an. nú-na < ${ }^{*} n \bar{u}-n \bar{e}$ y ${ }^{*} n u-n(e) H_{1}(e)-m$ (ai. nūnám, toc. A num, B nano, näno < * nu-nom) 'ahora'.

- De ser, como digo, un derivado, un pron. como gr. aủ-tós se diferencia del correspondiente adv. en ${ }^{*}-H_{1}-i /{ }^{*}-H-m$ sólo en que la base ${ }^{*} \mathrm{H}_{2} e u-t^{\mathrm{c}} \mathrm{o}_{\mathrm{o}}$ - recibe terminaciones propiamente flexionales.

- Podemos pensar (no para ${ }^{*} n \bar{u}$ ) que la relación de ambos elementos es la de un sintagma preposicional, es decir que el tema nominal regía sintácticamente al segundo elemento antes de la univerbación, y de ahí su propia capacidad de variación interna, por la que ${ }^{*}$ per $_{3}$ - puede aparecer como *per-, ${ }^{*}$ pr-, ${ }^{*}$ pro-, ${ }^{*}$ pre-, ${ }^{*} \mathrm{H}_{2} e u$ - como ${ }^{*} u-,{ }^{*} a u-,{ }^{*} u i-/$ *uī-, etc. En este caso, prons. como gr. aủ-rós se han formado por hipóstasis de la forma breve ${ }^{*} \mathrm{H}_{2} e u-t H$.

5.2.2. El segundo proceso de interés es la formación de compuestos propiamente dichos, de "gemelos" adverbiales. Lo llamaré tipo [PRON + FLEX] + [PRON + FLEX].

En este tipo el primer elemento suele portar como morfema flexivo un formante nasalizado ${ }^{*}-H-m$. El primer adv. puede ir en grado $\mathrm{P} \circ \emptyset$, sin que sea posible discernir una alternancia $\mathrm{P} / \varnothing, \varnothing / \mathrm{P}$ entre los dos advs. del compuesto. Como la mayoría de ejemplos se documenta en lenguas que no conservan diferencias en la cantidad del diptongo, no sabemos si tenemos en el primer adv. el tipo I (P/Ø) o II (Ø/P) del sufijo nasal y, como su distribución está fonotácticamente regulada y en nuestro caso el segundo adv. empieza por consonante, sólo es garantizable por comparación la antigüedad del compuesto.

El segundo término del compuesto es cualquier variante del adv. básico; en ocasiones tiene sufijo ${ }^{*}-H-m 0^{*}-H_{1}-i$ que son los tipos más frecuentes. Cf. en el primer caso lat. quondam, tandem, quandō, o. punum, quizá las dudosas aesl. kondon, lit. kandan-gi 'porque' ${ }^{22}$, aprus. isstwendan (que descenderían de un tipo balt.-esl. muy antiguo) y rus., etc. $k u d a$, tuda. Encontramos *- $H_{1}-i$ en germ. (aaa. hwande, aing. Yende- $n$, geond, giend, gót. jaind), y en aesl. kondẽ (si no es analógico), lat. inde (= lusit. indi 'y'), todos $<^{*}-d h(e) H_{1}-i$. Quizá se deba añadir a uno de los tipos galés pan 'cuando', airl. can '¿de dónde?'.

Es probable que ai. sada-, sádam y ai. sahá, aav., ap. hadā, nav.

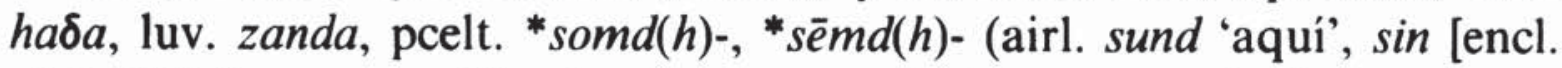
< 'alli']) hayan seguido el mismo procedimiento a partir de la reinterpretación coyuntural del tema nominal *sem/som/sm- 'uno' como adv.

${ }^{22}$ Citada por A. Bezzenberger, Beiträge zur Geschichte der litauischen Sprache, Gotinga 1877 , p. 40, como forma antigua de kadángi. 
pronominal. Este tema a su vez habrá contribuido a la selección casi exclusiva de las variantes ${ }^{*} k H o m /{ }^{*} k H m$ de ${ }^{*} k(e) H(e) m$ y a su incorporación, muchas veces como adv. comitativo, al uso preposicional, prefijal y preverbial, al que puede haber sido inicialmente ajeno el adv. pronominal.

La existencia del compuesto con segundo componente simple *-C-H está muy documentada, y es difícil distinguir el resultado, a veces, del tipo ADV + ENCL. Además aparece en balt.-esl. un tercer sufijo ${ }^{*}-d\left(h(e) H_{3}-u\right.$, que en esl. se extiende parcialmente a bases pronominales simples, pero quizá tiene en el compuesto su origen: cf. aprus. isquendau ¿desde dónde?', stwendau 'desde ahí; iswinadu 'fuera' (Enquir. 49, 34; ¿f. 1. por -dau?); aesl. kondu ‘¿de/por dónde?', rus. occ. kudy ‘¿adónde?', $\operatorname{kog} d y\left(<{ }^{*}-d \bar{u}\right)$. Ante la coincidencia de aparición de ${ }^{*}-d\left(h(e) H_{1}(e)-m\right.$ en aesl. y aprus. en las mismas bases que ${ }^{*}-d\left(h(e) H_{3}-u\right.$, puede pensarse incluso en un desarrollo balt.-esl. de éste.

El conjunto integrado por lat. ident-, lit. idañt, het. -idanta (infra 5.2.2 g) sigue un proceso normal de sufijación del tema pron. por un adv., como ocurre en PRON + [PRON + FLEX], aunque en este caso el adv. es un compuesto [PRON + FLEX] + [PRON + FLEX].

Dentro de este grupo hay que dedicar un subapartado a los advs. cuya base está caracterizada por ${ }^{*}-(e) H_{1}-i$ : se trata de gr. $\delta \varepsilon \tilde{i}-v a$, iva, instrs. ai. del tipo té-nā/na, tal vez los advs. germ. de base *hi-, a la que se podría atribuir un origen ${ }^{*} k H_{1}-i$. Les corresponden adjs. como gót.

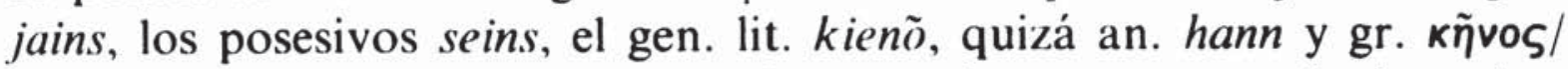
кEivos. Hay dos explicaciones: a) Se trata de compuestos de dos advs. en ${ }^{*}-H_{1}-i$ (cf. supra sobre la frecuencia del tipo ${ }^{*}-H-m+{ }^{*}-H-m$ ) o de ${ }^{*}-H-i+{ }^{*}-H-m$, según lenguas; la forma con segundo elemento «breve» ${ }^{*}-n H$, tal como aparece en gr. -va, se ha hipostasiado produciendo el tipo pronominal. b) Son derivados por medio de un sufijo ${ }^{*}-n^{\mathrm{e}} / \mathrm{o}^{-}$, constituidos en prons. por un lado, y que por otro reciben un final adv. *$H-m$ o *-H-i según tenga cada lengua en el tipo PRON + [PRON + FLEX] un suf. ${ }^{*} n(e)-H_{1}-m$ o ${ }^{*} n(e) H(e)-i$. Es un esquema [(PRON + FLEX) + SUF] + FLEX.

En todo caso, los casos oblicuos pron. het. con tema en diptongo como ked, kedi o kedani, kedanta no pertenecen a este tipo, sino que se han formado por una especie de hipóstasis inversa que permitió repetir de nuevo su mecanismo formacional: así, casos de PRON + [PRON + FLEX], como ${ }^{*} i-d h(e) H_{1}-i$ o ${ }^{*} i-d(e) H_{1}(e) m$, han perdido su independencia y han sufijado bases pron. het. comunes en una nueva aglutinación, definible como de segundo grado, PRON + [PRON + (PRON + FLEX)]. Existe duda razonable de si este proceso puede hacerse ex- 
tensivo a lat. quïdam. Schmidt (1988, pp. 124-25) reconstruye ${ }^{*} k^{u} o i-$ idām; pero una protoforma ${ }^{*} k^{u} o$-idam hubiera dado el mismo resultado.

5.2.3. Un tipo que no encaja entre los anteriores y que no ha sido estudiado es aquel por el que una sucesión de temas pron. yuxtapuestos recibe un morfema cuasi-flexivo que afecta a ambos sintácticamente, pero sólo al último de la serie formalmente. Es un proceso aún vivo en toc., donde en una sucesión de varios adjs. que determinan a un sust. en un caso secundario, sólo éste es portador de la marca de caso, y los adjs. van en oblicuo. Igualmente, en uná serie de susts., el último es el caracterizado por el caso secundario. En el sistema adverbial esto se entiende de dos formas:

a) Diacrónicamente hablando, una serie yuxtapuesta de dos o más temas pron. recibe un morfema cuasi-flexivo. Éste es, por así decir, el sistema "vivo", y esporádicamente afecta a advs. reduplicados como ${ }^{*} s(u) e H_{3^{-}}{ }^{*} s(u) e H_{3}-r$ en u. surur. Probablemente está en het. na-kku, ta$k k u$, donde es dificil hacer proceder $-k k u$ de ${ }^{*} k^{u} e$ - (existe en pal. y quizá lid.; el resultado normal deslabializado de ${ }^{*} k^{u} e$ está en el pron. het. $k u i s-k i)$. Así, reconstruyo ${ }^{*} t o-k^{u}(H) u^{23}$. El uso "coordinante» de $-k u$ en het. está muy mal representado; y el disyuntivo $-(a)$ - $k k u$ puede a su vez venir de ${ }^{*} o-k^{u}(H) u^{24}$. El mismo esquema aparece en $n a-s s u$, quizá = airl. nó, galés neu; en luv. nanun, alb. nani ${ }^{25}$, ai. nanú ‘¿acaso no?' (si hereda un esquema antiguo); het. ta-kiya, lit. ta-taĩ, ta-ciau, let. ta-su, lit. te-ciaũs, te-jaũ, let. te-ju, alb. këtu $\left(<{ }^{*} k^{u} o\right.$-tu $)$, toc. B tet $(e) k a \bar{a} k\left(<{ }^{*} t o-\right.$ to- $\left.{ }^{*} k^{u} \bar{o}\right)$, A $\breve{s} k \bar{a}$ / B ška $\left({ }^{*} k e-/{ }^{*} k^{u} e-{ }^{*} k^{(u)} \bar{o}\right)$, A škam $\left(<{ }^{*} k e-/{ }^{*} k^{u} e-k o m\right)$. Esto se formaliza como PRON + PRON + FLEX; puede que en algunos casos, desde el punto de vista diacrónico, tengamos en realidad un tema pron. seguido de adv. caracterizado, que confiere secundariamente al anterior su carácter flexivo.

b) Se distingue del tipo PRON + [PRON + FLEX] en que:

- En ocasiones se encuentra mera reduplicación de un mismo tema pronominal.

${ }^{23}$ Ven $-u$ en final, reconstruyendo a veces ${ }^{*} k u$ y sobre presupuestos morfológicos no siempre coincidentes con los nuestros, Sturtevant, A comparative grammar of the Hittite language, Filadelfia 1933, p. 120; Josephson, "Pronominal adverbs of Anatolian: formation and function", $R H A$ 24, 1966, p. 149; Hamp, "Celtica indogermanica», ZCPh 37, 1979, pp. 167-73, implícitamente Ivanov, "Syntactical Archaisms of Old Hittite", Hethitisch und Indogermanisch, 1979, pp. 76, al comparar aesl. takŭ.

${ }^{24} \mathrm{Y}$ no ${ }^{*} \mathrm{H}_{2} \mathrm{o}-k^{\psi} e$ paralelo a ${ }^{*} H_{2} u-k^{\psi} e$ como hace Dunkel, op. cit., p. 199.

2s $\mathrm{Si}$ *no-nü: E. Hamp, "Luwian nanun 'now'», RHA 27, 1969-70, pp. 132-33. 
- Sólo componen este tipo los temas pron. sintácticos ${ }^{*} i e-/^{*} i o-$, ${ }^{*} k^{u} e-/{ }^{*} k^{u} o-$ y ${ }^{*} t e /{ }^{*} t o-$, además de ${ }^{*} k e-/{ }^{*} k o$-; éste no existe como base ni como sufijo (excepto quizás en gr. -ka) en el esquema PRON + $[\mathrm{PRON}+\mathrm{FLEX}], \mathrm{y}^{*} k^{\mu} e-/{ }^{*} k^{\mu} o$ - no aparece en éste jamás como sufijo. No entran en juego los temas deícticos ${ }^{*}$ de-/*do-, ${ }^{*}$ dhe-/*dho-.

- En general, la combinación entre los dos o más temas es diferente de la de PRON + [PRON + FLEX], y parece más bien la condensación de los componentes de un díptico sintáctico.

- El sufijo ${ }^{*}-(e) \mathrm{H}_{3}$-u está ampliamente representado como caracterizador.

- Como en $[($ PRON + FLEX $)+$ SUF $]+$ FLEX, a veces existe junto al adv. PRON + PRON + FLEX un pron. completo, como sucede concretamente en balt.-esl., p. ej. en lit. kitas 'otro', šitas 'éste', tóks 'tal, tan', aesl. takü/jakŭ 'tal/cual', čìto 'qué', y alb. këta 'esto'.

- La independencia sintagmática relativa de ambos temas y su paridad funcional se evidencia en el hecho de que en la misma lengua aparecen advs. similares con caracterización doble y que pueden definirse como compuestos secundarios: cf. lit. tau-ciau, tuo-jaũ, let. kau-šu, toc. $t(a) k \bar{a}\left(<^{*} t u-k^{u} \bar{o}\right), y a k(a), y k \bar{a} k\left(<^{*} y u-k^{u} \bar{o}\right)$, y tal vez alb. te 'en'. Esta prep. acompaña singularmente al nom.; su variante dialectal es tek. Por ello Hamp (cf. n. 22) estima que, como te de ${ }^{*} t \bar{o}(i)$ o ${ }^{*} t o i$, tek procede de ${ }^{*} t \bar{o}(i){ }^{*}{ }^{*}{ }^{u} \bar{o}(i)$ o tal vez ${ }^{*} t o i-k u$, originalmente 'alli-donde (está) X', con verbo implícito; de hecho, tek se encuentra funcionando como 'alládonde' en subordinadas.

5.2.4. Existe, por lo demás, un interesante tipo mixto pronominalnominal en el que no es fácil adivinar la relación entre los términos. Es el integrado por gr. $\kappa\left\llcorner\delta v o ́ v<{ }^{*} k i\right.$-dinóm ${ }^{26}$, pgr. ${ }^{*} k i$-ämeron, seguramente reflejo formacional del anterior, pgr., palb. ${ }^{*} k i$-wetes y toc $\mathrm{B}$. ñerwe $<{ }^{*} n i-i \bar{e} r-$, tal vez frig. sest si $<{ }^{*} k i-$. Ante esto caben dos interpretaciones:

a) ${ }^{*} k i$ - ${ }^{*} n i$ - son temas pronominales, y sólo el tema nominal recibe una terminación que afecta a ambos, como en 5.2.3. En lenguas en que el tema se mantiene vivo como formador de prons., ambos términos se declinan y concuerdan normalmente. Así lit. siañ diẽn.

b) ${ }^{*} k i$ - y ${ }^{*} n i$ - son advs.; ello es plausible no sólo porque ${ }^{*} n i$ - realmente no puede ser otra cosa, sino porque la base del pron. secundariamente tematizado en balt.-esl. ${ }^{*} k i-{ }^{*} k i o-$ es seguramente un antiguo adv. Así parece confirmarlo el paralelismo *ki-dinom 'en el día de hoy':

${ }^{26}$ B. Prósper, "Über die Etymologie von gr. $\kappa\llcorner\delta v o ́ v », K Z$, en prensa. 
*peren-dinom 'en el día de pasado mañana' (lat. in perendinum). Un tipo paralelo a éste sería rus. tepérĭ, arus. topere, toc. A tāpär $(k)$ 'ahora', lat. topper y, diría yo, alb. tepër 'además' < *tō-per.

\subsection{Semántica}

He dicho en 4.4 que del conjunto PRON-FLEX se extrae en principio sólo una especificación sintáctica (demostr., interr., rel.) y una semántica/deíctica primaria (local/modal). También he indicado que la precisión direccional es ajena al esquema primario. Sin embargo, puede derivarse aquélla de las construcciones complejas.

a) En el tipo PRON + [PRON + FLEX] podemos esperar que el elemento PRON-FLEX haya conservado valores que ha perdido en situación autónoma: hay sentido local en ${ }^{*} i-d h(e) H_{1}-i,{ }^{*} i-d(e) H(e) m /$

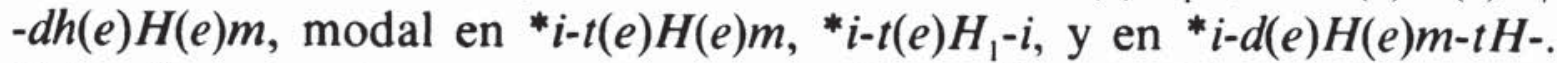
Esto afecta igualmente a sus correspondientes interrs. Es el sufijo el que determina el significado, la base pron. en cambio el empleo sintáctico como demostr., interr., etc.

b) En el tipo NOM + [PRON + FLEX] se ve un carácter marcadamente local de ${ }^{*}-t(e) H_{1}-i$, mientras que ${ }^{*}-t(e) H(e) m$ debe de haber sido modal en los casos en que alarga a ${ }^{*} \mathrm{H}_{2} e u$ - (ai. utá, lat. autem): es decir que ${ }^{*} H_{2} e u-t(e) H_{1}-i$ y ${ }^{*} H_{2} e u-t(e) H_{1}(e)-m$ pueden haber sido inicialmente advs. continuativos o adversativos de matiz respectivamente local y modal, hasta su confusión y reespecialización idiomática. * ${ }^{*} n(e) H_{1}-i /{ }^{*}$ $n(e) H(e) m$ son modales o de refuerzo en advs. locales (como en germ.); en tanto alarga a ${ }^{*} n u$ y ${ }^{*}$ per $H_{3}$ - el resultado es temporal, es decir que el tipo ${ }^{*}-n(e) H_{1}-i /{ }^{*}-n(e) H(e) m$ obra aquí probablemente sólo como refuerzo, dado que ambas bases tienen de por sí carácter temporal.

c) En el compuesto propiamente dicho [PRON + FLEX] + [PRON + FLEX] perviven tipos que a veces se hacen proceder de un adv. abl. Así ocurre en el tipo aprus. -en-dau, aesl. -on-du, lat. - $n$-de y germ. - $n-d-$, y quizás gr. ह̌v $\vartheta \varepsilon v$, que suelen remitirse a ${ }^{*}-n-d h-$. En los dos primeros, el valor abl. es inseguro por estar casi circunscrito en los temas ${ }^{*} k^{\mu} e-/{ }^{*} k^{u} o-$ y ${ }^{*} t e-/{ }^{*} t o-$ a usos con prep. Gr. ${ }^{*} v \vartheta \varepsilon v$ es un tipo mixto y debe dejarse al margen, y en $\pi \delta-\vartheta \varepsilon v$ el valor abl. es seguramente secundario.

Lat. y germ. tienen en comun un tema base ${ }^{*} i(e) H(e) m$-, germ. y balt.-esl. ${ }^{*} k^{\mu}(e) H(e) m /{ }^{*} t(e) H(e) m$, modales en casi todas las lenguas, y balt.-esl. y anat. (quizá celt.) ${ }^{*} k(e) H(e) m /{ }^{*} \operatorname{sem} / \mathrm{som}$. Ahora bien, de la 
adición a un adv. modal de una especificación local u orientador *-d(h)surge un adv. 'así pues, por ello' referido a un punto de la realidad, de donde lógicamente surge en ocasiones un adv. abl., y en otras el adv. deriva hacia el uso local. Este orientador puede haber sido tanto ${ }^{*}$-dhcomo ${ }^{*}$ - $d$ - en casi todos los casos; en el segundo, su función original sería anafórica. Es difícil decidir si la elección de un segundo término de compuesto ${ }^{*}-d\left(h(e) H_{1}-i\right)^{*} d(h)(e) H_{1}(e) m$ está o no condicionada a dos referencias deícticas a la misma realidad de matiz distinto.

En cambio no hay casos seguros en que el primer término de un

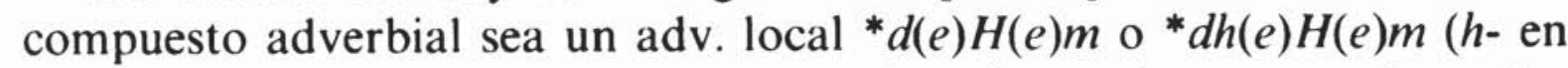
ai. hánta es ambigua). Lat. ident-, lit. idañt, het. -idanta son tal vez los únicos supervivientes de este esquema, ya no independientes, y tienen valor modal.

Si el segundo término es ${ }^{*} t-H$-, excepto en el subsistema local *-t(e) $H_{1}-i$, la confluencia de una base y un segundo término modales produce un adv. de la esfera modal. Así lat. quant-, tant-, arm. $k$ 'an (?) prons. cuantitativos, pal. -kuanta, u. -(h)ont (refuerzos encl.), quizá ai. hánta, an. hund- 'muy' (ide. ${ }^{*} k[H] m-t H$ puede pertenecer a ${ }^{*} t[e] H_{1}-i$ y $\left.{ }^{*} t[e] H[e] m\right)$, y lat. $i$-dent-, lit. $i$-dañt, het. -i-danta. Esto parece implicar que en general $-t$ pertenecía al subsistema modal * $t(e) H(e) m$, y la ausencia frecuente de nasalización del compuesto de dos advs. modales puede obedecer a una regla de no-redundancia, que viene a explicar parte de las "formas cortas" de sufijos con fonema laringal pero sin sonante, aunque en algunos casos ${ }^{*} t$-, etc., puede encubrir sólo un elemento enclítico puro.

d) En el tipo PRON + PRON + FLEX en cambio parece que ${ }^{*} t e-/{ }^{*} t o-,{ }^{*} k^{u} e-/{ }^{*} k^{u} o-,{ }^{*} i e-/{ }^{*} i o-\left(\right.$ tal vez $\left.{ }^{*} n e-/{ }^{*} n o-,{ }^{*} s[u] e-/{ }^{*} s[u] o-\right)$ incorporan exclusivamente contenido sintáctico: demostr.-demostr., demostr.-rel., indef.-demostr., donde la relación ha sido en ocasiones en su origen posiblemente de verdadera correlación, como en het. takku 'si', toc. B tetekāk, tetkāk 'de pronto, enseguida'. En otras, tal vez de formación analógica de este primer tipo, se yuxtaponen pleonásticamente elementos de contenido aproximado (cf. het. nassu), de los cuales va marcado el segundo.

Blanca Prósper 